\title{
Shaping by stiffening: a modeling study for lips
}

\author{
Mohammad A. NAZARI ${ }^{1,3}$, Pascal PERRIER ${ }^{1}$, Matthieu CHABANAS ${ }^{1}$, Yohan PAYAN ${ }^{2}$ \\ ${ }^{1}$ DPC/GIPSA lab, UMR CNRS 5216, Grenoble INP, France \\ ${ }^{2}$ TIMC-IMAG, UMR CNRS 5525, Université Joseph Fourier, Grenoble, France \\ ${ }^{3}$ Mechanical Engineering Depart. Faculty of Engineering, University of Tehran, Tehran, Iran
}

Emails: Mohammad.Nazari@gipsa-lab.grenoble-inp.fr

Pascal.Perrier@gipsa-lab.grenoble-inp.fr

Matthieu.Chabanas@gipsa-lab.grenoble-inp.fr

$\underline{\text { Yohan.Payan@imag.fr }}$

Motor Control, 15(1), 141-168

January 2011 


\section{ABSTRACT}

On the basis of simulations carried out with a finite element biomechanical model of the face, the influence of the muscle stress stiffening effect was studied for the protrusion/rounding of the lips produced with the Orbicularis Oris (OO). It is shown that the stress stiffening effect influences lip shape. When stress stiffening is modelled, the variation in the crucial geometrical characteristics of the lips shows a clear saturation effect as the OO activation level increases. Similarly, for a sufficient amount of $\mathrm{OO}$ activation, a saturation effect is observed when stiffening increases. In both cases, differences in lip shaping associated with the absence or presence of stiffening have consequences for the spectral characteristics of the speech signal obtained for the French vowel /u/. These results are interpreted in terms of their consequences for the motor control strategies underlying the protrusion/rounding gesture in speech production.

Key words: speech motor control, stiffness, biomechanics, orofacial muscles, soft tissues, lip shape 


\section{Introduction}

Stiffness properties of the human motor system depend on various physiological influences, such as passive elastic properties of muscle tissues, muscle activations, and neural feedback (McMahon, 1984). Thus muscle activations in motor systems not only induce changes in position but also changes in stiffness. Stiffness changes and position changes intrinsically co-occur as the consequences of muscle activation, but to a certain extent they can also be controlled separately. Evidence supporting the hypothesis of these separate controls has been well documented in different studies that have shown the existence of (1) isometric motor tasks (change in muscle activations and stiffening, but no change in position), (2) isotonic motor tasks (change in position and in individual muscle activations, but without change in global muscle activation), and (3) unconstrained motor tasks (change in position and in muscle activations and stiffening) (see for example Feldman, 1986, for an account of these separate controls in the context of the Equilibrium Point Hypothesis). According to the literature, a specific control of stiffness is useful in motor control mainly for two reasons: increasing stiffness has an impact on movement speed and duration, and it is an efficient way to control movement accuracy, especially in the context of external perturbations.

A number of experimental (Bennett, 1993; Suzuki et al., 2001) and modelling studies (Gribble \& Ostry, 1998; Perrier et al., 1996) of speech production have shown that high stiffness levels of the motor system are associated with rapid movements. Controlling the stiffness of the motor system allows the global movement duration for a given movement amplitude to be influenced. In a pure dynamical representation of motor systems (Kelso, 1995) the motor system is represented as sets of coupled oscillators. In the framework of coupled oscillators it has been proposed that modulating stiffness would be the means to control time, in the form of individual movement durations as well as of interlimb (or, for speech, interarticulator) time coordination (phasing) (Kelso et al., 1981; Kelso, 1995, p.104-106; for speech production see also Saltzman, 1986). For speech research this proposal is grounded in the intrinsic speech timing theory proposed by Fowler (1980).

So far, the majority of recent studies on stiffness control in movement production are related to the question of movement accuracy control. It has been shown that, for postural control, increasing the co- 
activation of agonist and antagonist muscles would minimize the consequences of perturbing external loads by increasing joint stiffness (see for example Milner \& Cloutier, 1998, and Milner, 2002 for the wrist joint). Similar findings have been obtained for movement control in perturbed conditions. For example, Burdet et al. (2001) observed that, in a motor task where the subjects had to follow with their arm a straight line connecting two targets, the subjects adapted to an unstable velocity-dependent perturbing force field imposed by a robot by increasing the stiffness of their arm in the direction of a perturbing field. However, as suggested by Milner (2002), increasing the stiffness is "metabolically costly" (p. 406). Hence, it can be expected that under normal movement execution, i.e. in the absence of a perturbing field, subjects could favour alternative and less costly motor strategies to ensure accuracy. Milner \& Franklin (2005) indeed found that, in the presence of a perturbing force field, subjects would increase stiffness only during the first trial. According to these authors, for the other trials, other strategies were elaborated based on the acquisition of a crude internal model of the dynamics in the perturbed conditions. Assuming the acquisition and the use of internal dynamical representations, similar results were observed by Davidson \& Wolpert (2003) for subjects performing a single task in a predictably varying environment. A number of studies confirm, though, that even under normal, unperturbed conditions, stiffness is used to control accuracy. Studying jaw movements in speech production with respect to relations between positioning accuracy and stiffness ellipses, Shiller et al. (2002) found that accuracy is better in the direction of the major axes of the ellipses, i.e. movements are more precise in the directions along which stiffness is larger. Osu et al. (2004) observed that in tasks where subjects were asked to reach targets of different sizes, they stiffened their arm when the targets were small. These authors also noticed that movements intentionally produced with increased co-contraction were more accurate. Similarly, Wong et al. (2009) measured the variation in limb stiffness when subjects were asked to move their arm from a start target to an end target, in the absence of any perturbing external force field, with various requirements in terms of end target accuracy. The authors observed that stiffness increased when the size of the end target decreased.

Hence, the relation between stiffness of the limb, the jaw or the arm and movement accuracy seems to be well established. However, all motor effectors studied so far are rigid bodies articulated at joints. 
Stiffening these effectors actually means stiffening the joints, by increasing the forces of the agonistantagonist sets of muscles (a coordinated muscle activation also called co-activation) around these joints. In the case of speech production, the articulators that are responsible for the fine shaping of the vocal tract, which determines the properties of the acoustic signals, are soft bodies such as the tongue, lips, and velum. The impact of stiffening on soft bodies is likely to be quite different from that observed for rigid bodies. Indeed, stiffening a joint changes the stability of the motor system around this joint, without modifying in any way the range of the configurations that are likely to be achieved by the motor system. In the case of rigid bodies a change in stiffness modifies the dynamical characteristics of the task space, but it does not alter the shape or the size of the task space. For soft bodies, the situation is different because different stiffness values may affect the required shape of motor system. Consequently, it can be expected that controlling stiffness could be used in speech movements for other purposes than in limb or arm movements.

We are aware of only one study on the role of stiffening in the control of soft body motor systems. It was proposed by Hooper (2006) and concerns the octopus. Hooper (2006) explains the observation that octopuses stiffen their tentacles when they grasp at food by the fact that this strategy allows a reduction in the number of degrees of freedom of the tentacle. This reduction simplifies the selection of the motor commands that enable a precise achievement of a particular gesture. However, to our knowledge, there is so far no study of the role of stiffening in soft body motor control for humans. In this paper we propose a study of the potential effects of stiffening in lips' movements. Our approach consists in developing and using a biomechanical model of the lips. This was deliberately chosen rather than an experimental study with humans, because it allows a quantitative specific assessment of stiffening, which would be difficult in experimental conditions. The interpretation of our results should allow the design of experimental studies in the future aimed at testing the hypotheses inferred from our simulations.

\section{Method: Model}

Many physically based models of the human face have been developed in the framework of computer graphics for facial animation (Lee et al., 1995; Lucero and Munhall, 1999; Sifakis et al., 2005), 
computer aided surgery (Chabanas et al., 2003; Gladilin et al., 2004) and speech production study (Gomi et al., 2006; Kim \& Gomi, 2007). The pioneering work by Lee et al. (1995) has made discrete modelling frameworks popular, where sparse mass-spring entities are regularly assembled inside facial tissues. This approach allows fast computation with a simple algorithmic implementation. However, in addition to the lack of accuracy of such models and to their numerical instabilities, it seems to be very difficult to set their elastic parameters (the stiffness of springs) in order to model the constitutive behaviour that is measured on living tissues. Recently Kim \& Gomi (2007) have improved Gomi et al.'s (2006) model by implementing a so-called "continuum compatible" mass-spring model with stiffness parameters that can be adjusted in order to be compatible with continuum mechanics and with a simple linear continuum constitutive law. However, since human tissues behave non-linearly (Fung, 1993; Gérard et al., 2005) Kim \& Gomi’s (2007) model cannot reproduce realistic physical behaviour. For this reason, as a continuation of the model developed by Chabanas et al. (2003), we have chosen the finite element method to model the continuous behaviour of human facial tissues.

Our 3D finite element model is implemented in ANSYS (R) release 11.0 software and consists of three parts: (1) the main part is a mesh that models the facial tissues; (2) cable elements are attached to the mesh to implement muscle forces; and (3) contact elements are added to formulate muscle/passive tissue coupling and inter-lip and lip/teeth collision. It has been extensively described in Nazari et al. (2010). Only the main features necessary to assess the reliability of the simulations will be presented below.

\section{Face mesh: Geometry and mechanical properties}

The main mesh is a finite element approximation of the volume representing facial tissues between the skull and the external facial surface. Anatomically, face tissues consist of epidermis, dermis and hypodermis (Standring, 2005). Most face muscles are flat muscles and are contained in the hypodermis layer. The mesh has three distinct layers, which makes it suitable to facilitate the implementation of potentially different mechanical properties in future work. The current version of the mesh is composed of 6342 brick elements, in the form of both hexahedral and wedge shapes

(Figure 1). The mesh is symmetrical with respect to the mid-sagittal plane. The dimensions of the 
mesh in directions perpendicular to the sagittal, transverse, and coronal planes respectively are: 144 $\mathrm{mm}, 128 \mathrm{~mm}$, and $138 \mathrm{~mm}$.

Figure 1 around here

The outer and inner surfaces of the mesh were extracted from CT scans of an adult subject. Then the mesh was semi-automatically constructed using brick elements. The elements do not have mid-side nodes, so the total number of nodes is 8736 . Material properties of elements are assumed to follow a 5parameter Mooney-Rivlin hyperelastic model (Fung, 1993). This non-linear elastic model is determined by a strain-energy function $W$, whose derivative with respect to strain gives stress $\left(S_{i j}=2 \partial W / \partial C_{i j}, S_{i j}\right.$ are components of the second Piola-Kirchhoff stress tensor, and $C_{i j}$ are components of the right Cauchy-Green deformation tensor):

$W=c_{10}\left(I_{1}-3\right)+c_{01}\left(I_{2}-3\right)+c_{20}\left(I_{1}-3\right)^{2}+c_{11}\left(I_{1}-3\right)\left(I_{2}-3\right)+c_{02}\left(I_{2}-3\right)^{2}+\left((J-1)^{2} / d\right)$

where $c_{n m}$ are the parameters of the model, $I_{1}$ and $I_{2}$ are respectively the first and second invariants of the right Cauchy-Green strain tensor, $J$ is the determinant of the elastic deformation gradient, and $d=(1-2 v) /\left(c_{10}+c_{01}\right)$, where $v$ is the Poisson's ratio. Similar to Gérard et al. (2005), in our model a simplified version is used in which only two constants, $c_{10}$ and $c_{20}$, are non-zero. According to Tracqui \& Ohayon (2004), $c_{10}$ can be approximated by the formula $c_{10} \approx E / 6$, where $E$ is the Young's modulus. The two coefficients $c_{10}$ and $d$ have been calculated from the values $E=15 \mathrm{kPa}$ and $v=0.499$ proposed for facial tissues in Chabanas et al. (2003) with the assumption of mechanical linearity and tissue incompressibility. As compared to the value proposed by Gérard et al. (2005) for the tongue, the $c_{10}$ coefficient is 13 times larger, because of differences in the Young's modulus. Hence, to determine $c_{20}$ we multiplied Gérard et al.'s $c_{20}$ value with the same factor. The computed constants are shown in Table 1.

Table 1 around here

The mechanical properties of specific elements of a muscle are modified with the activation of the muscle to model the stress stiffening effect during activation using a method that will be explained below. 


\section{Muscle arrangement}

Ten orofacial muscles (Standring, 2005) are modelled, which are listed together with their corresponding abbreviations in Table 2. Since a detailed description of all muscle fibers would require too much complexity, muscle forces are applied to the mesh via macrofibers that account for the main muscle fiber directions. The approach is consistent with the design of other speech articulator models (the jaw, Laboissière et al., 1996, and the tongue, Payan \& Perrier, 1997; Gérard et al., 2006; Buchaillard et al., 2006; Buchaillard et al., 2009). The macrofiber directions were extracted from CT scans based on anatomical knowledge (Standring, 2005) with the help of a maxillofacial surgeon. The number of macrofibers per muscle depends on the extent and size of each muscle (see Figure 2).

Figure 2 around here

Table 2 around here

Macrofibers are modelled with 3D cable elements which are two-node line elements that can apply only compressive force on the tissues to which they are connected. Since a cable element is a straight line in space, the curved geometry of a macrofiber is approximated by a piecewise set of elements. The number of cable elements increases as a function of muscle length and fiber curvature, in order to model the muscle smoothly. When they are stretched, these elements generate compressive forces that linearly increase as a function of muscle length.

Cable elements are connected to the mesh via coupling contact elements (see below).

\section{Boundary Conditions}

\section{Contact Elements}

Our model contains two groups of contact elements. The first group couples cable elements with the main mesh. The second group models the actual contact surfaces, between lips and teeth or between upper and lower lips. 
The insertions and the orientations of the cable elements were determined according to anatomical data. They are independent of the element definition of the main mesh. To link these cable elements with the main mesh, point to surface contact elements are used. The points, called pilot nodes, are extremities of the cable elements, and the corresponding surfaces are the external surfaces of the brick elements whose centroid is near to those extremities. In Figure 3 cable elements and corresponding coupling contact elements are shown for the OOP and ZYG major.

Figure 3 around here

For modelling inter-lip and lip/teeth contacts, surface to surface contact elements are used (Figure 4). The surfaces of teeth on mandible and maxilla are approximated with spline surfaces (Figure 4b) and are discretized with quadrilateral rigid elements. The surface contact elements have no initial interpenetration and are realized without friction.

------ Figure $4 \mathrm{a}$ and $4 \mathrm{~b}$ around here

\section{Fixed-Point Boundary Conditions}

Since in our current model the jaw is not mobile, the nodes of the surface modelling the teeth are fixed. The inner nodes of the internal layer of the main face mesh, which correspond to facial tissue attachments to the skull, are also fixed. The extremities of cable elements corresponding to muscle insertions on the skull are fixed as well.

\section{Modelling Muscle Activation}

The muscle force is divided into an active and a passive part. In our model, the active part is represented by the action of the cable elements, while the passive part is represented by the intrinsic elastic properties of the main face mesh. To simulate active muscle force generation, a control parameter $T$ is used that determines the force produced by the cable elements. When $T$ decreases, the cable element length starts decreasing and therefore, like in real muscles, the cable element exerts force on the main mesh through the coupling contact elements. Starting from a zero force configuration a parameter difference $\Delta T$ will induce a force: 


$$
F=K(\varepsilon-\alpha \Delta T)
$$

where $K$ is a gain factor, $\varepsilon$ is the engineering strain, and $\alpha$ is a tuning parameter. $K$ and $\alpha$ have no physiological or physical meaning, but are scaling parameters to boost the level of force. They are chosen to ensure numerical convergence while generating displacements of realistic amplitudes.

The increase in muscle stiffness associated with muscle activation is functionally modelled following the same approach as proposed by Buchaillard et al. (2006). This functional change is modelled by changing the coefficients of the constitutive law of the elements that are assumed to represent activated muscle tissues in the main mesh. These elements are determined by a spherical neighbourhood algorithm that selects the elements of the main mesh which are included in a sphere around the cable elements. The radius of the sphere varies with the muscle of interest. For example, Figure 5 shows the elements that correspond to the OOP and the ZYG major muscles. The stiffness properties of these elements change proportionally to activation $(T)$, and they are computed by scaling the $c_{10}$ and $c_{20}$ coefficients of the Mooney-Rivlin constitutive law within a range from 1 to 10 (Figure 6) (for more details, see Nazari et al., 2010).

------- Figure 5 around here

--------Figure 6 around here --------

\section{Simulations and measures}

Static and full transient analyses were carried out in order to test the realism of the model's behaviour in response to various muscle activation patterns. In addition to the impact of tissue elasticity, the transient analysis takes into account the effect of inertia and gravity as well as the effect of viscosity. Special attention is devoted in this paper to lip protrusion and lip rounding gestures, which are basic speech gestures associated with the phonetic characteristics "rounded" versus "spread" lips (IPA Handbook, IPA, 1999). Classically these gestures are analysed in the literature as being generated mainly with the activation of the Orbicularis Oris (OO) (Delaire, 1977; Abry et al., 1980; Standring, 2005). So far, to our knowledge, biomechanical models have not been successful in modelling lip rounding in a realistic way. To study the potential contribution of muscle stiffening to the achievement of this gesture, 
simulations were run with and without the changes in the constitutive law associated with muscle activation.

Variability of lip shaping due to the different simulated conditions is measured using the parameters proposed by Abry \& Boë (1986). A description of these parameters in profile view and frontal view is shown in Figures 7 and 8 respectively. They measure the amplitude of the protrusion (lip horn depth, lip corner protrusion, upper lip protrusion and lower lip protrusion), the aperture (lip opening, lip height, lip area) and the rounding (ratio between lip height and lip width). These three geometrical features are basic means to characterize labial speech gestures. They are measured between the rest position and the stable shape attained at the end of the movement simulation.

\section{------- Figure 7 around here -------- \\ -------- Figure 8 around here --------}

In the presented simulations, the activation parameter $T$ is incremented as a staircase function of the time, so approximates a linear variation. Since we want to focus on the lip shape at the end of the movement and not on its time variation during the movement, this simple approach of staircase activation seems appropriate. According to the modelling of the stress stiffening effect, the increase of parameter $T$ generates a variation in the parameters $c_{10}$ and $c_{20}$ of the constitutive law. Different amplitudes of the $T$ variation were tested in the simulations: $c_{10}$ and $c_{20}$ are multiplied by a factor ranging from 1 (no change in stiffness) to 10 (strong increase in stiffness). Hence, in our simulations two degrees of freedom are manipulated independently: the amplitude of the global change in $T$ and the global increase in stiffening. This approach enables the influence of two factors to be determined independently: the amplitude of the activation and the amplitude of the associated stiffening. It is important to clarify that this approach was set up in order to study the response of the model to different activation/stiffening conditions, and not in order to account for any physiological process in which stiffening and activation would be decoupled. 


\section{Results}

In all tested cases, transient analysis converged. No numerical instabilities were observed. It can be concluded that the constitutive law, the mass and the viscosity factor used in the model correspond to a stable dynamical system. In addition, the lip shapes obtained at the end of the simulations based on transient analysis are very similar to those obtained with static analysis. Hence, since transient analysis is very demanding in terms of computation time, and since in this paper we do not address any movement related issue but are only interested in the final shaping, the simulations presented below were run in the static context.

As stated above, the presentation of the results focuses on the role of stress stiffening in the achievement of rounded lips. The production of some vowels (called rounded vowels, as opposed to spread vowels) requires a small lip area. Classic examples of this vowel category are the French vowels $/ \mathrm{u} /, / \mathrm{y} /$ or $/ \varnothing /$. For a large majority of subjects, this small area is achieved by protruding the lips, in spite of the fact that the protrusion gesture is not the only way to achieve a small lip area. This regularity across speakers is interpreted as evidence for the fact that protrusion is an efficient way to achieve small lip areas. Our simulations, in the context of the effect of stiffening on shaping, aim at further understanding this strategy. The impact of the Orbicularis Oris (OO) activation is analyzed with and without the stress stiffening effect.

The presentation of the results is organized in two main subsections. First we study the impact of the OO activation and of the stress stiffening on the lip shape based on the parameters proposed by Abry \& Boë (1986). Second, an evaluation of the impact of the different simulated lip shapes on the spectral properties of the associated acoustic speech signal is performed on the French vowel /u/. In both studies, the role of the stress stiffening effect is at the core of the analysis.

\section{Lip protrusion and rounding gesture}

Figure 9 shows the final lip shape obtained with our face model for the activation of the OOP associated with the largest stiffening value (multiplication factor of $c_{10}$ and $c_{20}$ is equal to 10). 
To observe the effect of stiffening the same simulation was run with the same activation level and the same timing, but without accounting for the stress stiffening effect (multiplication factor of $c_{10}$ and $c_{20}$ is equal to 1) (Figure 10). As compared to Figure 1 (rest position), both figures show a clear protrusion and a reduction in lip area. This reduction is less strong though without stiffening, mainly because the lip height is significantly larger. The lip shape achieved without stiffening does not correspond to a prototypical protruded/rounded shape, mainly because of this large lip height value.

Figure 10 around here

Two preliminary conclusions can be drawn from these first qualitative observations: (1) in our model the Orbicularis Oris Peripheralis simultaneously generates a protrusion and a narrowing of the lips; (2) accounting for the stress stiffening effect significantly influences lip shaping and it reinforces the efficiency of the protrusion gesture in achieving a small lip area, which are the key characteristics of rounded lips. In order to further assess these preliminary conclusions and to do so quantitatively, four sets of simulations were run. In two sets (Table 3) the stress stiffening level varied in 10 regular steps from its minimum (factor equal to 1 ) to its maximum (factor equal to 10) while the activation level was set either to its minimum (Table 3a) or to its maximum (Table 3b). In two other sets of simulations (Table 4), the activation level varied in twelve regular steps from its minimum (0.1) to its maximum (0.4) while the stress stiffening level was set either to its minimum (Table 4a) or to its maximum (Table $4 b)$.

-----Table $3 \mathrm{a}$ and $3 \mathrm{~b}$ around here-----

Table $4 \mathrm{a}$ and $4 \mathrm{~b}$ around here

The results of Tables $3 \mathrm{a}$ and $3 \mathrm{~b}$ are depicted in Figures $11 \mathrm{a}$ and $11 \mathrm{~b}$ respectively. These figures show that for both levels of muscle activation, while the majority of the geometrical lip parameters tend to globally vary linearly with the increase in stress stiffening, the lip area and the lip height follow a clear non-linear variation. This non-linearity is characterized by a rapid decrease for low stress stiffening 
levels followed by a quasi-stabilization above a certain stress stiffening level (around 4 for low activation and 6 for high activation). This corresponds to a saturation effect. For the high activation level, lip horn also shows such a saturation effect, but this is not true for the low activation level. Interestingly the two parameters that depict a saturation effect independently of the activation level, namely the lip area and the lip height, are considered to be crucial for the geometrical characterization of lip shapes in the protrusion/rounding condition (Abry \& Boë, 1986). In terms of motor control, this saturation effect is interesting since it enables the same values for crucial lip shape parameters to be reached over a wide range of motor commands.

Figure $11 \mathrm{a}$ and $11 \mathrm{~b}$ around here

Would a control of the activation amplitude be as efficient as the stiffening? To answer this question, the next two sets of simulations are very informative. The results, listed in Tables $4 \mathrm{a}$ and $4 \mathrm{~b}$, are plotted respectively in Figures 12a (no stiffening) and 12b (maximal stiffening). In the absence of stiffening (Figure 12a) all the measured lip characteristics vary linearly with the activation level. No saturation is observed, even for high activation levels. For the maximum stress stiffening level (Figure 12b) clear saturation effects are observed above the activation level 0.3 for 4 parameters, lip area, lip height, lip width and lip horn depth. In addition, the decrease in the lip area reached for a given level of activation is much larger with stiffening than in the absence of stiffening. When the stress stiffening effect is taken into account, an increase in activation is associated with a decrease in lip height. This behaviour is consistent with a prototypical protrusion/rounding gesture. In contrast, when the stress stiffening effect is not modelled, increasing the activation generates an increase in lip height, a result which is in opposition to the characteristics of a lip protrusion/rounding gesture. These results are in complete agreement with the impressionistic conclusions made from Figures 9 and 10. The reduction in lip area without a saturation effect and with an increase in lip height observed in the non-stiffening conditions suggests that modelling the stress stiffening effect is extremely positive for an efficient control of lip shaping, especially regarding the rounding associated with protrusion. The increase in the activation level is "as efficient as the stiffening" only if stiffening is taken into account. The effect of stiffening on lip protrusion/rounding is confirmed. 
The realism or absence of realism of the different accounts of the protrusion/rounding gesture given in the different simulation conditions can be quantitatively assessed thanks to the experimental observations provided by Abry \& Boë (1986). These authors present (see Figure 3 of their paper) a law that describes how the lip area (S), lip height (B), and lip width $(\mathrm{A})$ are linked with each other: $\mathrm{S}=$ $0.75^{*} \mathrm{~A} * \mathrm{~B}$. An additional way to evaluate our results is to test whether one of the simulation conditions gives results compatible with Abry \& Boë's experimental findings. Figure 13 presents the results of this evaluation. In Figure 13a, the dashed line shows the lip area function that would result from our lip height and width measurements according to Abry \& Boë's law while the solid line corresponds to the measured area function. Figure $13 \mathrm{~b}$ shows the ratio between the calculated and the measured area. It can be observed that in the absence of stiffening, the ratio is significantly larger than 1. Hence, the simulated lip shapes do not match the experimental observations. This ratio decreases dramatically as soon as stiffening is taken into account. When stiffening increases, the ratio continues to decrease, almost linearly, with the multiplying factor, but significantly less strongly. The ratio becomes very close to one when the multiplying factor is equal to 10. This shows that with the inclusion of stiffening, the relation between lip parameters is in agreement with the experimental observations. Thus, including stiffening enables the generation of more natural lip shapes.

Figure $13 \mathrm{a}$ and $13 \mathrm{~b}$ around here

\section{Impact of the stress stiffening effect on the spectral properties of the acoustic speech}

signal: The example of the vowel /u/

To study the effect of stiffening on the frequency content of the speech signal, we generated synthetic speech waves from a vocal tract in which the lip characteristics were those generated with our model in both stiffened and non-stiffened cases. The vocal tract geometry corresponded to the French vowel $/ \mathrm{u} /$, which is a classic example of a rounded vowel. For this purpose we took the data proposed for this vowel in Figure 1 of Apostol et al. (2004). Then we replaced the geometrical characteristics of the sections corresponding to the lips with the data extracted from our model. The addition of lip corner 
protrusion to lip horn depth ( $\mathrm{L}$ in Figure 7) defines the length of the lip tube, and the lip area (S in Figure 8) is used for the cross-section of this tube. As an example an area function is show in Figure 14a. This area function is then used as an input function for a Kelly-Lochbaum digital speech synthesis model (Kelly \& Lochbaum, 1962; Story, 2005). The speech signal generated with this model (Story, 2005) is then analysed in the spectral domain. With the help of the Linear Prediction Analysis, the spectral envelope of this signal is extracted (Figure 14b). The spectral peaks correspond to formants, which are crucial for the perceptual quality of the vowels. For the French vowel /u/ the key spectral characteristic is that the first two formants F1 and F2 are in the low frequency domain $[300 \mathrm{~Hz} 700$ Hz]. Savariaux et al. (1999, Figure 3) show that the perceptual quality of this vowel improves when F2 decreases within this range to become closer to F1. In a Standard French $/ \mathrm{u} /$, such as the one modelled in Apostol et al. (2004), F1 mainly depends on the geometry of the back part of the vocal tract and F2 is mainly influenced by the front part and by the lips. The analysis of F1-F2 narrowing, and especially of the F2 decrease, thus provides a suitable basis to investigate acoustically the effect of stiffening in the modelled protrusion/rounding gesture.

-------Figure $14 \mathrm{a}$ and $14 \mathrm{~b}$ around here--------

The variation in the first two formants is extracted for different lip shape parameters corresponding to an increase in the stiffness level (with minimum and maximum activation levels) (Figure 15a) and to an increase in the activation level (with minimum and maximum stiffening values) (Figure 15b).

\footnotetext{
---------Figure 15a and 15b around here---------
}

As Figure 15a shows, when the stress stiffening effect is modelled, while the activation level is kept at its maximum (bold line), the second formant decreases and converges towards the first formant, which remains fairly constant. This F2 decrease is consistent with a more rounded and protruded lip shape. It is not observed for the minimum activation level (solid line). Figure $15 \mathrm{~b}$ shows that an increase in the activation level induces a decrease in F2 only if it is accompanied by stiffening (bold line). In the absence of stiffening (solid line) increasing activation has no spectral consequences. 
Thus, the acoustic simulation suggests that the association of muscle activation increase and stiffening enables the production of more canonical spectral patterns for the French vowel $/ \mathrm{u} /$.

\section{Discussion and Conclusion}

On the basis of simulations carried out with a finite element biomechanical model of the face, the impact of the stress stiffening effect (i.e. of the tissue stiffening associated with muscle activation) was studied for the protrusion/rounding gesture of the lips. The lips' protrusion/rounding gesture was generated by activating the upper and lower parts of the OOP. It was found that the stress stiffening effect significantly influences shaping. Acoustic simulations showed that the differences in lip shaping corresponded to differences in spectral patterns.

Stiffening in the lip protrusion/rounding gesture significantly changes the shape of the lips: in the absence of stiffening, protrusion is produced and is associated with a reduction in the lip area, but the achieved lip shape does not match experimental data on rounding because of the lip height. Protrusion is associated with a clear reduction in lip height only if stiffening is taken into account. Hence, in the case of the lips, it can be concluded that stiffening is useful for shaping. This finding could be one explanation for the fact that, to our knowledge, the protrusion/rounding gesture has never been achieved with biomechanical models with the activation of the Orbicularis Oris alone (see for example the analysis proposed by Gomi et al. (2006) of their own simulation results).

In addition, our simulations have shown that when the stress stiffening effect is modelled, a saturation effect exists when the activation level increases. From a motor control perspective, this result is very interesting since it suggests that a simple strategy to generate protruded and rounded lips could be to activate the Orbicularis Oris while stiffening the tissues.

Another important result is that for a sufficient amount of activation of the Orbicularis Oris, increasing stiffening also generates a saturation effect in the variation in the crucial geometrical characteristics of the lips. This observation raises the following question: would it be possible to control stiffening in the Orbicularis Oris Peripheralis? In the literature the classic way to increase stiffness corresponds to a coordinated increase in the agonist and antagonist muscle activations. In our simulations of lip 
protrusion/rounding, only the Orbicularis Oris Peripheralis is activated. This muscle consists of two parts, the Orbicularis Oris Peripheralis Superior (OOPS) and the Orbicularis Oris Peripheralis Inferior (OOPI), which actually collaborate to generate protrusion and rounding, while exerting quasiantagonist forces on the lip corner. Hence, even if it does not correspond to the classic agonist/antagonist case, simultaneously changing the force generated by the OOPS and the OOPI seems to be an appropriate way to control stiffening. In this context, it can be concluded that controlling the intensity of the stress stiffening effect of the $\mathrm{OO}$ via the control of its activation could be an efficient strategy to accurately achieve lips' protrusion and rounding.

\section{References}

Abry, C., Boë, L.J., Corsi, P., Descout, R., Gentil, M. \& Graillot, P. (1980). Labialité et Phonétique. Publications de l’Université des Langues et Lettres de Grenoble, Grenoble, France.

Abry, C., \& Boë, L. (1986). “Laws” for lips. Speech Communication, 5, 97-104.

Apostol, L., Perrier, P., \& Bailly, G. (2004). A model of acoustic interspeaker variability based on the concept of formant-cavity affiliation. J. Accoust. Soc. Am., 115(1), 337-351.

Bennett, D.J. (1993). Torques generated at the human elbow joint in response to constant position errors imposed during voluntary movements. Experimental Brain Research, 95, 488-498.

Buchaillard, S., Perrier, P. \& Payan, Y. (2006). A 3D biomechanical vocal tract model to study speech production control: How to take into account the gravity? Proceedings of the $7^{\text {th }}$ International Seminar on Speech Production, 403-410.

Buchaillard S., Perrier P. \& Payan Y. (2009). A biomechanical model of cardinal vowel production: Muscle activations and the impact of gravity on tongue positioning. Journal of Acoustical Society of America, 26(4), 2033-2051.

Burdet, E., Osu, R., Franklin, D.W., Milner, T.E., \& Kawato, M. (2001). The central nervous system stabilizes unstable dynamics by learning optimal impedance, Nature, 414, 446-449. 
Chabanas, M., Luboz, V., \& Payan, Y. (2003). Patient specific finite element model of the face soft tissues for computer-assisted maxillofacial surgery. Medical Image Analysis, 7, 131-151.

Davidson, P.R. \& Wolpert, D.M. (2003). Motor learning and prediction in a variable environment. Current Opinion in Neurobiology, 13, 1-6.

Delaire, J., Fève, J. R., Chateau, J.P., Courtay, D., \& Tulasne, J.F. (1977). Anatomie et Physiologie des muscles et du frein médian de la lèvre supérieure. Premiers résultats de l'électromyographie sélective. Revue de Stomatologie, 2, 93-103.

Feldman, A.G. (1986). Once more on the Equilibrium-Point hypothesis ( $\lambda$ model) for motor control. Journal of Motor Behavior, 18, 17-54.

Fowler, C. A. (1980)._Coarticulation and theories of extrinsic timing control. Journal of Phonetics, 8 , 113-133.

Fung, Y.C. (1993). Biomechanics: Mechanical properties of living tissues. Springer-Verlag New York Inc.

Gérard, J.M., Ohayon, J., Luboz, V., Perrier, P., \& Payan, Y. (2005). Non-linear elastic properties of the lingual and facial tissues assessed by indentation technique, Application to the biomechanics of speech production. Medical Engineering \& Physics, 27, 884-892.

Gladilin, E., Ivanov, A., \& Roginsky, V. (2004). A framework for biomechanical simulation of cranio-maxillofacial surgery interventions. In Cotin, S., Metaxas, D. (Eds.), Proceedings of the ISMS 2004, 287-294.

Gomi, H., Nozoe, J., Dang, J., \& Honda, K. (2006). A physiologically based model of perioral dynamics for various lip deformations in speech articulation. In Harrington, J., Tabain, M. (Eds.), Speech Production: Models, Phonetic Processes, and Techniques (pp 119-134), Psychology Press.

Gribble, P.L., \& Ostry, D.J. (1998). Independent coactivation of shoulder and elbow muscles. Experimental Brain Research, 123, 355-360

Hooper, S. (2006). Motor control: The importance of stiffness. Current Biology, 16, 283-285.

IPA (1999) Handbook of the International Phonetic Association. Cambridge: Cambridge University Press. 
Kelly, J.L. \& Lochbaum, C.C. (1962). Speech Synthesis. Proceedings of the Fourth Int. Congress on Acoustics, Copenhagen, Denmark '62, 1-4.

Kelso, J.A.S. (1995). Dynamic Patterns: the self-organization of brain and behavior. MIT Press, Cambridge, Massachusetts.

Kelso, J.A.S., Holt, K.G., Rubin, P., \& Kugler, P.N. (1981). Patterns of human interlimb coordination emerge from the properties of non-linear oscillatory processes: Theory and data. Journal of Motor Behavior, 13, 226-261.

Kim, K., \& Gomi, H. (2007). Model-based investigation of control and dynamics in human articulatory motion. Journal of System Design and Dynamics, 1, 558-569.

Laboissière, R., Ostry, D.J., \& Feldman, A.G. (1996). The control of multi-muscle systems: human jaw and hyoid movements. Biological Cybernetics, 74, 373-384.

Lee, Y., Terzopoulos, D., \& Waters, K. (1995). Realistic modelling for facial animation. In Mair, S.G., Cook, R. (Eds.), Proceddings of the SIGGRAPH'95 55-62.

Lucero, J.C., \& Munhall, K.G. (1999). A model of facial biomechanics for speech production. Journal of Acoustical Society of America, 106, 2834-2842.

McMahon, T.A. (1984). Muscles, Reflexes, and Locomotion. Princeton University Press, Princeton, N.J.

Milner, T.E. (2002). Adaptation to destabilizing dynamics by means of muscle cocontraction. Experimental Brain Research, 143, 406-416.

Milner, T.E., \& Cloutier, C. (1998). Damping of the wrist joint during voluntary movement. Experimental Brain Research, 122, 309-317.

Milner, T.E., Franklin, D.W. (2005). Impedance control and internal model use during the initial stage of adaptation to novel dynamics in humans. Journal of Physiology, 567.2, 651-664.

Nazari, M.A. , Perrier, P., Chabanas, M., \& Payan, Y. (2010) Simulation of dynamic orofacial movements using a constitutive law varying with muscle activation, Computer Methods in Biomechanics and Biomedical Engineering, 13(4), 469 - 482 
Osu, R., Kamimura, N., Iwasaki, H., Nakano, E., Harris, C.M., Wada, Y., \& Kawato, M. (2004). Optimal impedance control for task achievement in the presence of signal-dependent noise. Journal of Neurophysiology, 92, 1199-1215.

Payan, Y. \& Perrier, P. (1997). Synthesis of V-V sequences with a 2D biomechanical tongue model controlled by the Equilibrium Point Hypothesis. Speech Communication, 22, 185-205.

Perrier, P., Lœvenbruck, H. \& Payan, Y. (1996). Control of tongue movements in speech: The Equilibrium Point Hypothesis perspective. Journal of Phonetics, 24, 53-75.

Saltzman, E. (1986). Task dynamic coordination of the speech articulators: A preliminary model. In Heuer, H. \& Fromm, C. (Eds.), Experimental Brain Research Series 15 (pp. 129-144). SpringerVerlag, New York.

Savariaux C., Perrier P., Orliaguet J.P. \& Schwartz J.L. (1999). Compensation strategies for the perturbation of French $[\mathrm{u}]$ using a lip tube. II. Perceptual analysis. Journal of the Acoustical Society of America, 106 (1), 381-393.

Shiller, D.M., Laboissière, R., \& Ostry, D.J. (2002). The relationship between jaw stiffness and kinematic variability in speech. Journal of Neurophysiology, 88, 2329-2340.

Sifakis, E., Neverov, I., \& Fedkiw, R. (2005). Automatic Determination of Facial Muscle Activations from Sparse Motion Capture Marker Data. ACM Transactions on Graphics (SIGGRAPH Proceedings), TOG 24, 417-425.

Standring, S. (editor in chief) (2005). Gray's Anatomy: The Anatomical Basis of Clinical Practice, $39^{\text {th }}$ Edition, Elsevier Ltd.

Story, B. H. (2005). A parametric model of the vocal tract area function for vowel and consonant simulation. J. Accoust. Soc. Am., 117(5), 3231-3254.

Suzuki, M., Shiller, D.M., Gribble, P.L., Ostry, D. J. (2001). Relationship between cocontraction, movement kinematics and phasic muscle activity in single-joint arm movement. Experimental Brain Research, 140, 171-181.

Tracqui, P. \& Ohayon, J. (2004). Transmission of mechanical stresses within the cytoskeleton of adherent cells: a theoretical analysis based on a multi-component model. Acta Biotheoretica, 52, $323-341$ 
Wong, J., Wilson, E. T., Malfait, N., \& Gribble, P. L. (2009). Limb stiffness is modulated with spatial accuracy requirements during movement in the absence of destabilizing forces, Journal of Neurophysiology, 101, 1542-1549. 
Table 1- Constants of simplified 5-parameter Mooney-Rivlin model for inactive muscle.

\begin{tabular}{|l|l|l|}
\hline$c_{10}(\mathrm{Mpa})$ & $c_{20}(\mathrm{Mpa})$ & $d(1 / \mathrm{Mpa})$ \\
\hline $2.5 \mathrm{e}-3$ & $1.175 \mathrm{e}-3$ & 0.8 \\
\hline
\end{tabular}


Table 2- Orofacial muscles for half of face.

\begin{tabular}{|c|c|}
\hline Muscle Name & Abbreviation \\
\hline $\begin{array}{c}\text { Levator Labii Superioris } \\
\text { Alaeque Nasi }\end{array}$ & LLSAN \\
\hline Levator Anguli Oris & LAO \\
\hline Zygomaticus (major and minor) & ZYG \\
\hline Risorius & RIS \\
\hline Buccinator & BUC \\
\hline Depressor Anguli Oris & DAO \\
\hline Depressor Labii Inferioris & DLI \\
\hline Mentalis & OONT \\
\hline $\begin{array}{c}\text { Orbicularis Oris Peripheralis } \\
\text { (Inferioris and Superioris) }\end{array}$ & OOM \\
\hline $\begin{array}{c}\text { Orbicularis Oris Marginalis } \\
\text { (Inferioris and Superioris) }\end{array}$ & \\
\hline
\end{tabular}


Table 3a- Different lip parameters with respect to different stiffness levels at minimum activation

\begin{tabular}{|r|r|l|l|l|l|l|r|r|r|}
\hline Stiffness & $\begin{array}{l}\text { CORNER LIP } \\
\text { PROTRUSION } \\
(\mathrm{mm})\end{array}$ & $\begin{array}{l}\text { LIP } \\
\text { HORN } \\
\text { DEPTH } \\
(\mathrm{mm})\end{array}$ & $\begin{array}{l}\text { LIP AREA } \\
\left(\mathrm{mm}^{2}\right)\end{array}$ & $\begin{array}{l}\text { LIP } \\
\text { HEIGHT } \\
(\mathrm{mm})\end{array}$ & $\begin{array}{l}\text { LIP } \\
\text { WIDTH } \\
(\mathrm{mm})\end{array}$ & $\begin{array}{l}\text { LIP } \\
\text { OPENING } \\
(\mathrm{mm})\end{array}$ & $\begin{array}{l}\text { LOWER LIP } \\
\text { PROTRUSION } \\
(\mathrm{mm})\end{array}$ & $\begin{array}{l}\text { UPPER LIP } \\
\text { PROTRUSION } \\
(\mathrm{mm})\end{array}$ & $\begin{array}{l}\text { Maximum } \\
\text { Force (N) }\end{array}$ \\
\hline 1 & 7.08 & 8.29 & 23.98 & 2.52 & 14.00 & 16.93 & 3.35 & 4.30 & 2.39 \\
\hline 2 & 6.61 & 8.55 & 20.23 & 2.15 & 13.77 & 15.73 & 3.07 & 3.58 & 2.79 \\
\hline 3 & 6.22 & 8.73 & 19.19 & 1.96 & 14.10 & 15.09 & 2.87 & 3.20 & 3.16 \\
\hline 4 & 5.84 & 8.17 & 18.85 & 1.87 & 14.44 & 14.64 & 2.70 & 3.03 & 3.41 \\
\hline 5 & 5.51 & 8.32 & 18.75 & 1.82 & 14.66 & 14.32 & 2.56 & 2.76 & 3.57 \\
\hline 6 & 5.21 & 8.72 & 19.39 & 1.83 & 15.61 & 14.10 & 2.48 & 2.57 & 3.75 \\
\hline 7 & 4.94 & 8.88 & 19.31 & 1.81 & 15.65 & 13.92 & 2.38 & 2.44 & 3.88 \\
\hline 8 & 4.69 & 9.04 & 19.36 & 1.80 & 15.88 & 13.76 & 2.29 & 2.34 & 3.95 \\
\hline 9 & 4.47 & 9.19 & 19.36 & 1.76 & 15.84 & 13.62 & 2.22 & 2.27 & 4.04 \\
\hline 10 & 4.30 & 9.31 & 19.46 & 1.75 & 15.96 & 13.51 & 2.15 & 2.21 & 3.91 \\
\hline
\end{tabular}


Table 3b- Different lip parameters with respect to different stiffness levels at maximum activation

\begin{tabular}{|r|r|r|r|r|r|r|r|r|r|}
\hline Stiffness & $\begin{array}{l}\text { CORNER LIP } \\
\text { PROTRUSION } \\
(\mathbf{m m})\end{array}$ & $\begin{array}{l}\text { LIP } \\
\text { HORN } \\
\text { DEPTH } \\
(\mathbf{m m})\end{array}$ & $\begin{array}{l}\text { LIP AREA } \\
\left(\mathbf{m m}^{2}\right)\end{array}$ & $\begin{array}{l}\text { LIP } \\
\text { HEIGHT } \\
(\mathbf{m m})\end{array}$ & $\begin{array}{l}\text { LIP } \\
\text { WIDTH } \\
(\mathbf{m m})\end{array}$ & $\begin{array}{l}\text { LIP } \\
\text { OPENING } \\
(\mathbf{m m})\end{array}$ & $\begin{array}{l}\text { LOWER LIP } \\
\text { PROTRUSION } \\
(\mathbf{m m})\end{array}$ & $\begin{array}{l}\text { UPPER LIP } \\
\text { PROTRUSION } \\
(\mathbf{m m})\end{array}$ & $\begin{array}{l}\text { Maximum } \\
\text { Force (N) }\end{array}$ \\
\hline 1 & 9.77 & 6.58 & 20.39 & 3.07 & 10.78 & 18.99 & 5.33 & 7.38 & 4.65 \\
\hline 2 & 10.19 & 7.32 & 17.70 & 2.59 & 10.52 & 17.90 & 5.25 & 6.97 & 5.67 \\
\hline 3 & 10.17 & 7.63 & 15.27 & 2.14 & 10.59 & 17.09 & 5.12 & 6.53 & 6.40 \\
\hline 4 & 10.07 & 7.58 & 13.47 & 1.84 & 10.76 & 16.48 & 4.98 & 6.24 & 6.95 \\
\hline 5 & 9.97 & 7.59 & 12.06 & 1.62 & 10.89 & 16.07 & 4.85 & 5.88 & 7.53 \\
\hline 6 & 9.85 & 7.61 & 11.13 & 1.46 & 11.00 & 15.73 & 4.75 & 5.71 & 8.13 \\
\hline 7 & 9.79 & 7.63 & 11.23 & 1.44 & 11.07 & 15.58 & 4.63 & 5.59 & 8.61 \\
\hline 8 & 9.64 & 7.67 & 10.65 & 1.35 & 11.16 & 15.32 & 4.53 & 5.40 & 9.20 \\
\hline 9 & 9.48 & 7.73 & 10.38 & 1.29 & 11.21 & 15.13 & 4.43 & 5.25 & 9.64 \\
\hline 10 & 9.35 & 7.78 & 10.23 & 1.25 & 11.28 & 14.96 & 4.34 & 5.12 & 10.00 \\
\hline
\end{tabular}


Table 4a- Different lip parameters with respect to different activation levels at minimum stiffness

\begin{tabular}{|r|r|r|r|r|r|r|r|r|r|}
\hline & $\begin{array}{l}\text { CORNER LIP } \\
\text { PROTRUSION } \\
(\mathrm{mm})\end{array}$ & $\begin{array}{l}\text { LIP } \\
\text { HORN } \\
\text { AETtivation } \\
(\mathrm{mm})\end{array}$ & $\begin{array}{l}\text { LIP AREA } \\
\left(\mathrm{mm}^{2}\right)\end{array}$ & $\begin{array}{l}\text { LIP } \\
\text { HEIGHT } \\
(\mathrm{mm})\end{array}$ & $\begin{array}{l}\text { LIP } \\
\text { WIDTH } \\
(\mathrm{mm})\end{array}$ & $\begin{array}{l}\text { LIP } \\
\text { OPENING } \\
(\mathrm{mm})\end{array}$ & $\begin{array}{l}\text { LOWER LIP } \\
\text { PROTRUSION } \\
(\mathrm{mm})\end{array}$ & $\begin{array}{l}\text { UPPER LIP } \\
\text { PROTRUSION } \\
(\mathrm{mm})\end{array}$ & $\begin{array}{l}\text { Maximum } \\
\text { Force (N) }\end{array}$ \\
\hline 0.1 & 7.08 & 8.29 & 23.98 & 2.52 & 14.00 & 16.93 & 3.35 & 4.30 & 2.39 \\
\hline 0.125 & 7.67 & 8.14 & 23.55 & 2.58 & 13.62 & 17.29 & 3.68 & 4.86 & 2.72 \\
\hline 0.15 & 8.20 & 7.97 & 23.11 & 2.62 & 13.44 & 17.58 & 3.94 & 5.38 & 3.05 \\
\hline 0.175 & 8.72 & 7.82 & 22.76 & 2.69 & 13.10 & 17.89 & 4.25 & 5.93 & 3.42 \\
\hline 0.2 & 9.06 & 7.70 & 22.39 & 2.72 & 13.06 & 18.06 & 4.39 & 6.23 & 3.67 \\
\hline 0.225 & 9.34 & 7.66 & 22.57 & 2.89 & 12.50 & 18.38 & 4.64 & 6.53 & 3.90 \\
\hline 0.25 & 9.45 & 7.64 & 22.21 & 2.95 & 12.53 & 18.46 & 4.75 & 6.67 & 4.03 \\
\hline 0.275 & 9.70 & 7.57 & 21.43 & 2.98 & 11.93 & 18.64 & 4.93 & 6.96 & 4.28 \\
\hline 0.3 & 9.59 & 7.09 & 20.65 & 3.04 & 11.45 & 18.86 & 5.18 & 7.19 & 4.42 \\
\hline 0.325 & 9.85 & 6.96 & 21.34 & 3.06 & 11.78 & 18.83 & 5.08 & 7.16 & 4.42 \\
\hline 0.35 & 9.90 & 6.93 & 21.51 & 3.12 & 11.66 & 18.87 & 5.12 & 7.22 & 4.47 \\
\hline 0.38 & 9.77 & 6.58 & 20.39 & 3.07 & 10.78 & 18.99 & 5.33 & 7.38 & 4.65 \\
\hline 0.4 & 10.25 & 6.57 & 18.97 & 3.15 & 10.82 & 19.37 & 5.69 & 7.96 & 5.22 \\
\hline
\end{tabular}


Table 4b- Different lip parameters with respect to different activation levels at maximum stiffness

\begin{tabular}{|r|r|r|r|r|r|r|r|r|r|}
\hline & $\begin{array}{l}\text { CORNER LIP } \\
\text { PROTRUSION } \\
(\mathrm{mm})\end{array}$ & $\begin{array}{l}\text { LIP } \\
\text { HORN } \\
\text { DEPTH } \\
(\mathrm{mm})\end{array}$ & $\begin{array}{l}\text { LIP AREA } \\
\left(\mathrm{mm}^{2}\right)\end{array}$ & $\begin{array}{l}\text { LIP } \\
\text { HEIGHT } \\
(\mathrm{mm})\end{array}$ & $\begin{array}{l}\text { LIP } \\
\text { WIDTH } \\
(\mathrm{mm})\end{array}$ & $\begin{array}{l}\text { LIP } \\
\text { OPENING } \\
(\mathrm{mm})\end{array}$ & $\begin{array}{l}\text { LOWER LIP } \\
\text { PROTRUSION } \\
(\mathrm{mm})\end{array}$ & $\begin{array}{l}\text { UPPER LIP } \\
\text { PROTRUSION } \\
(\mathrm{mm})\end{array}$ & $\begin{array}{l}\text { Maximum } \\
\text { Force (N) }\end{array}$ \\
\hline 0.1 & 4.30 & 9.31 & 19.46 & 1.75 & 15.96 & 13.51 & 2.15 & 2.21 & 3.91 \\
\hline 0.125 & 5.05 & 9.63 & 17.37 & 1.65 & 15.61 & 13.63 & 2.47 & 2.58 & 4.68 \\
\hline 0.15 & 5.73 & 8.68 & 15.54 & 1.52 & 14.48 & 13.74 & 2.76 & 2.86 & 5.54 \\
\hline 0.175 & 6.46 & 8.36 & 13.66 & 1.43 & 13.50 & 13.88 & 3.06 & 3.26 & 6.37 \\
\hline 0.2 & 6.90 & 8.19 & 12.47 & 1.31 & 13.32 & 13.99 & 3.26 & 3.53 & 6.92 \\
\hline 0.225 & 7.48 & 7.94 & 11.49 & 1.26 & 11.88 & 14.16 & 3.51 & 3.91 & 7.58 \\
\hline 0.25 & 7.80 & 7.78 & 10.92 & 1.22 & 11.78 & 14.25 & 3.65 & 4.12 & 8.02 \\
\hline 0.275 & 8.25 & 7.53 & 10.33 & 1.19 & 11.65 & 14.42 & 3.86 & 4.47 & 8.60 \\
\hline 0.3 & 8.68 & 7.97 & 9.93 & 1.19 & 11.49 & 14.60 & 4.06 & 4.78 & 9.09 \\
\hline 0.325 & 8.73 & 7.94 & 9.75 & 1.17 & 11.47 & 14.61 & 4.09 & 4.81 & 9.27 \\
\hline 0.35 & 9.01 & 7.86 & 10.50 & 1.26 & 11.40 & 14.82 & 4.16 & 5.08 & 9.52 \\
\hline 0.38 & 9.35 & 7.78 & 10.23 & 1.25 & 11.28 & 14.96 & 4.34 & 5.12 & 10.00 \\
\hline 0.4 & 9.89 & 7.59 & 9.83 & 1.24 & 11.09 & 15.18 & 4.64 & 5.53 & 10.89 \\
\hline
\end{tabular}




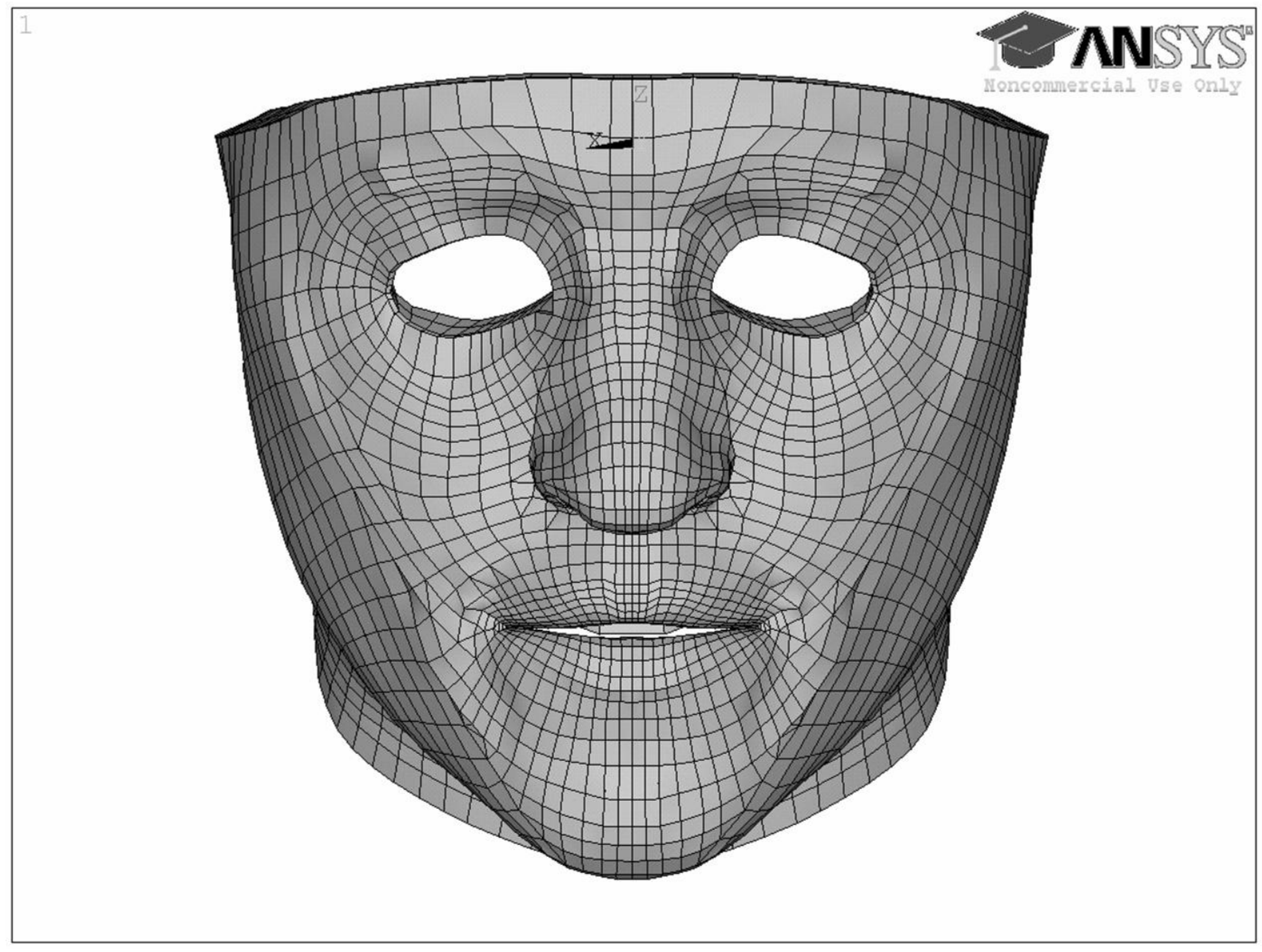

Figure 1- Main mesh. 


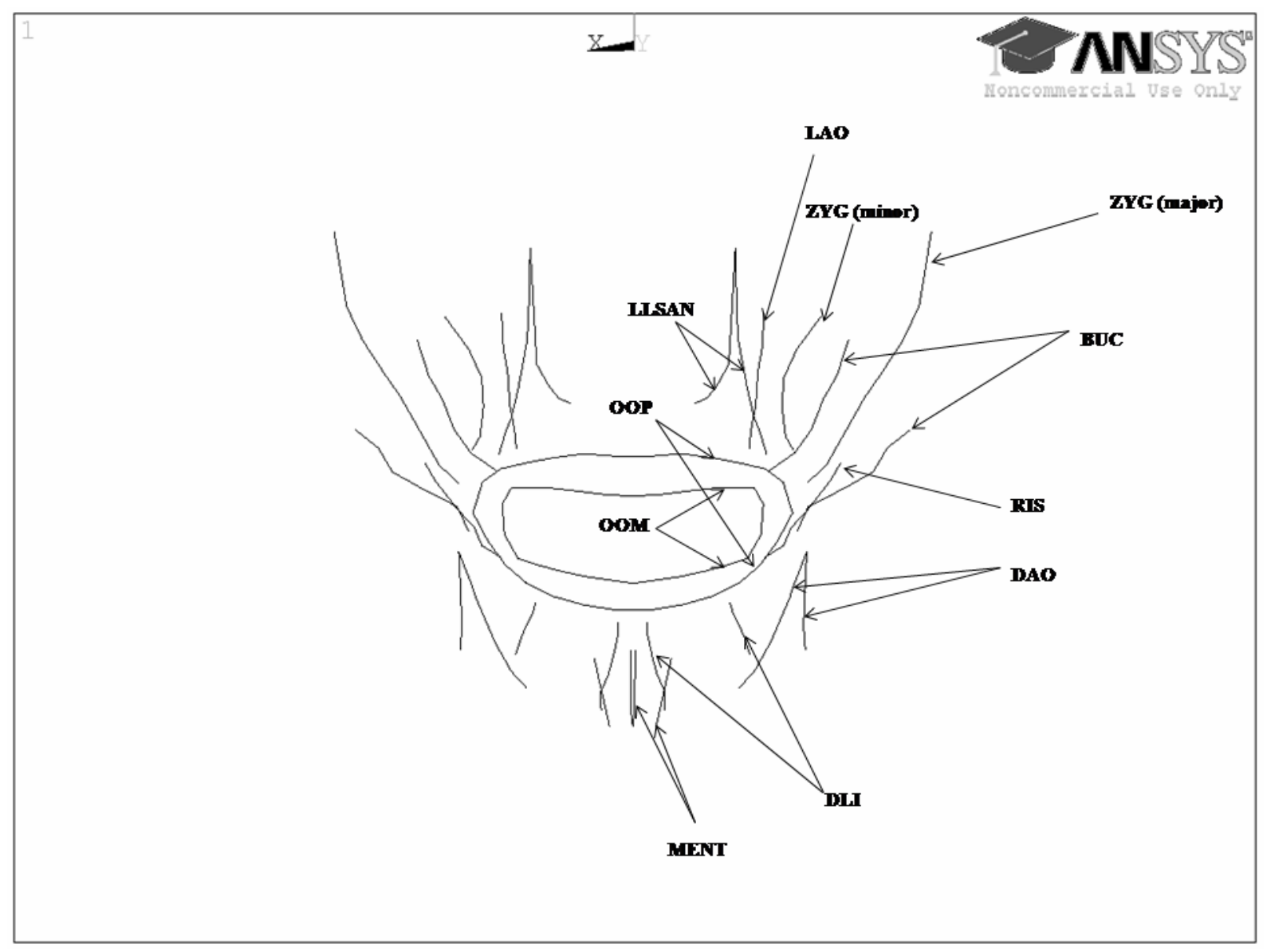

Figure 2- Macrofibers of the ten modelled muscles. 


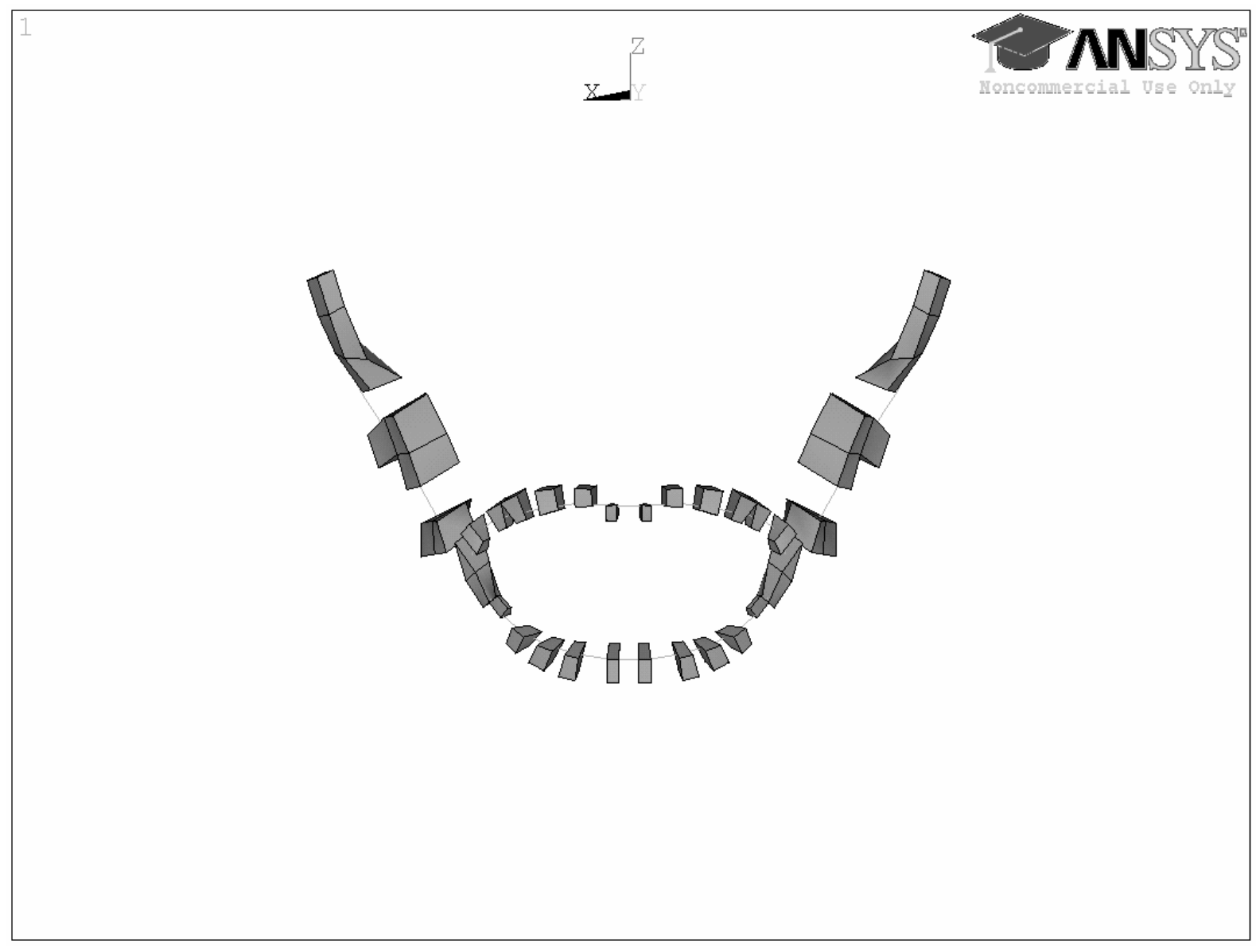

Figure 3- Coupling contact elements for the OOP and ZYG major. 


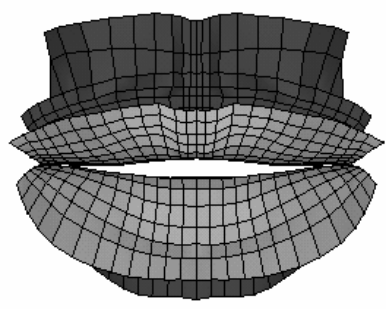

(a)

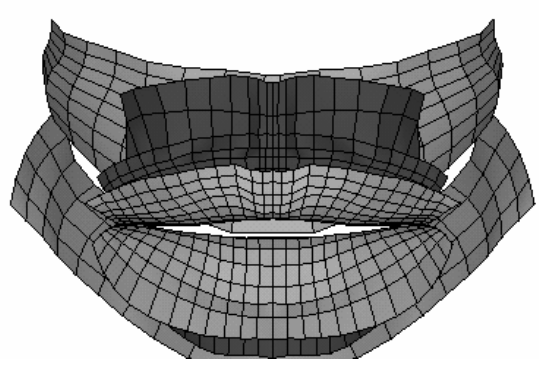

(b)

Figure 4- Surface contact elements between lips (a) and lips and teeth (b). 


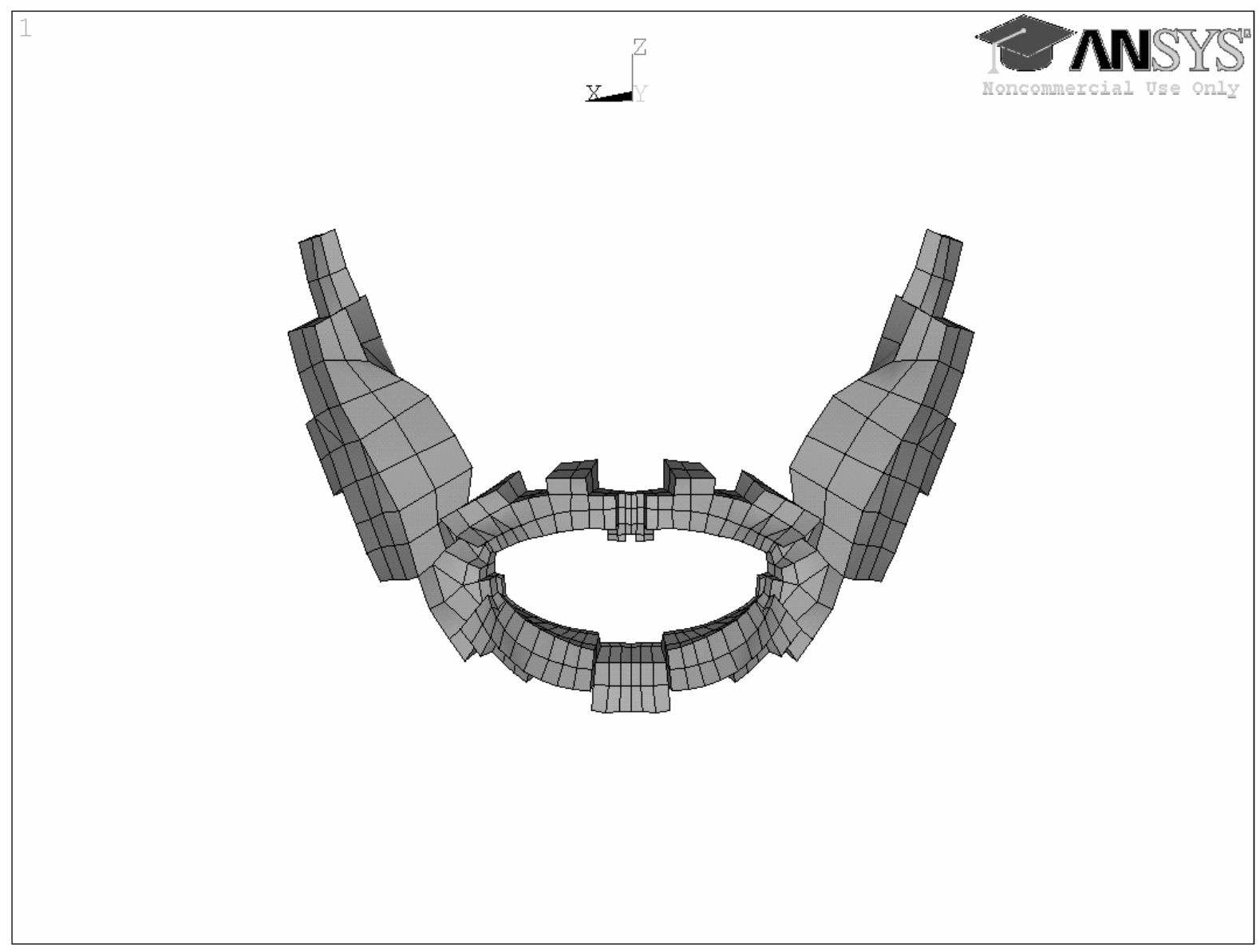

Figure 5- Muscle elements from the main mesh corresponding to the ZYG major and the OOP muscles as selected with the spherical neighbourhood algorithm (see text) with a radius of 2 and 3 $\mathrm{mm}$ respectively. 


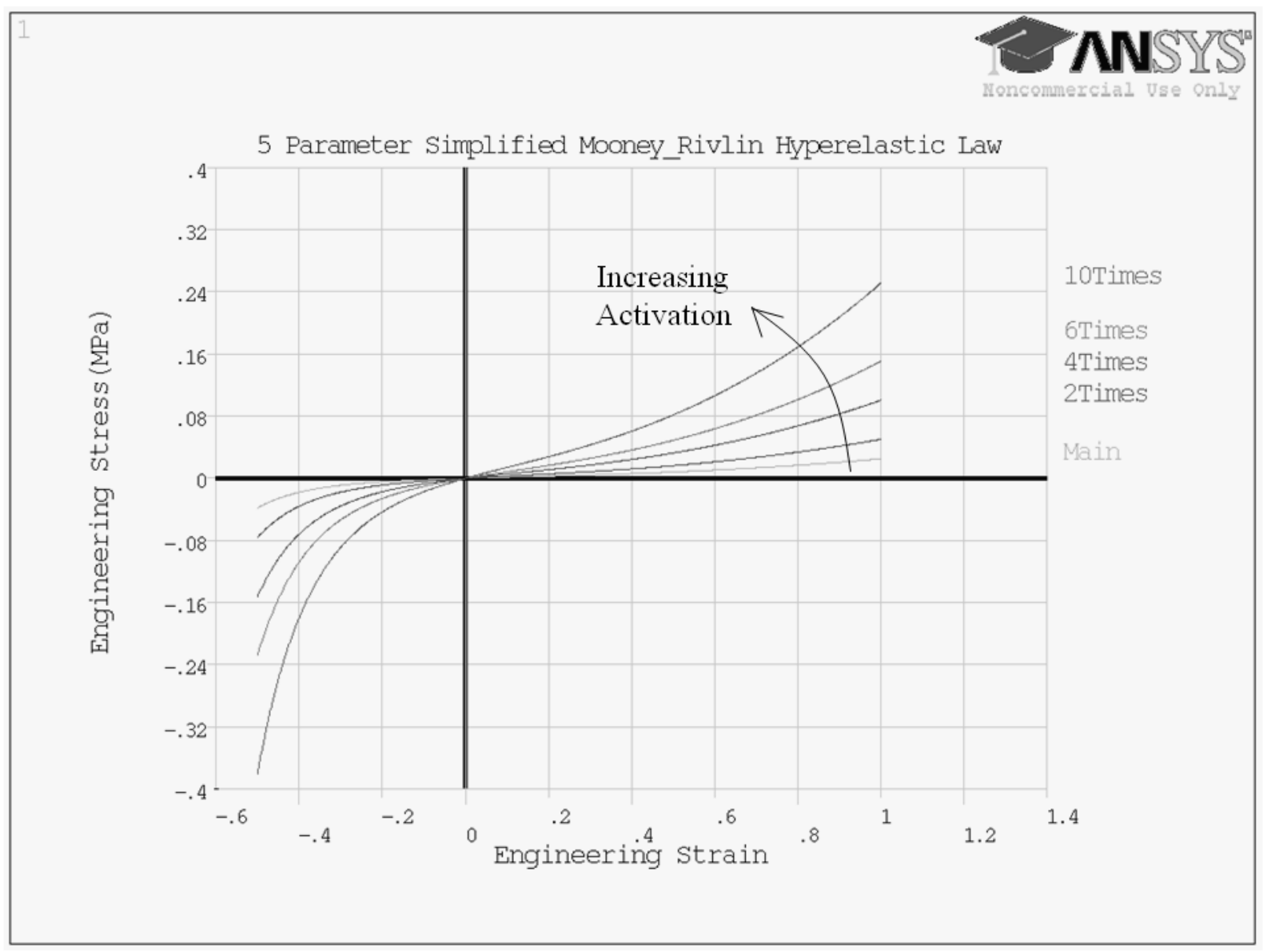

Figure 6- Variation of the constitutive law functionally accounting for the stress stiffening effect associated with muscle activation. 


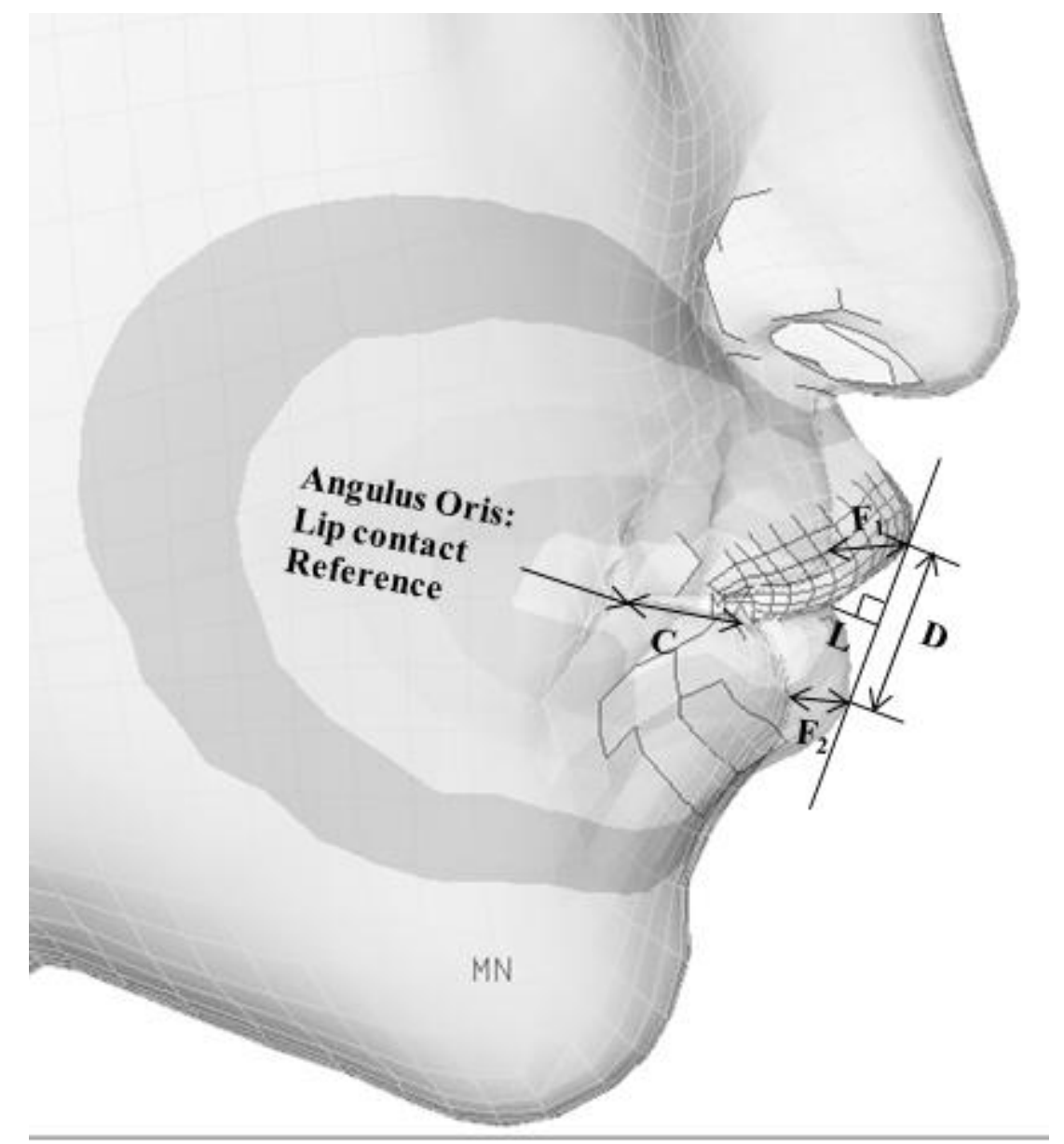

Figure 7- Lip shape parameters (profile view): lip opening (D), lip horn depth (L), lip corner protrusion $(\mathrm{C})$, upper lip protrusion $\left(\mathrm{F}_{1}\right)$, lower lip protrusion $\left(\mathrm{F}_{2}\right)($ Abry \& Boë, 1986). 


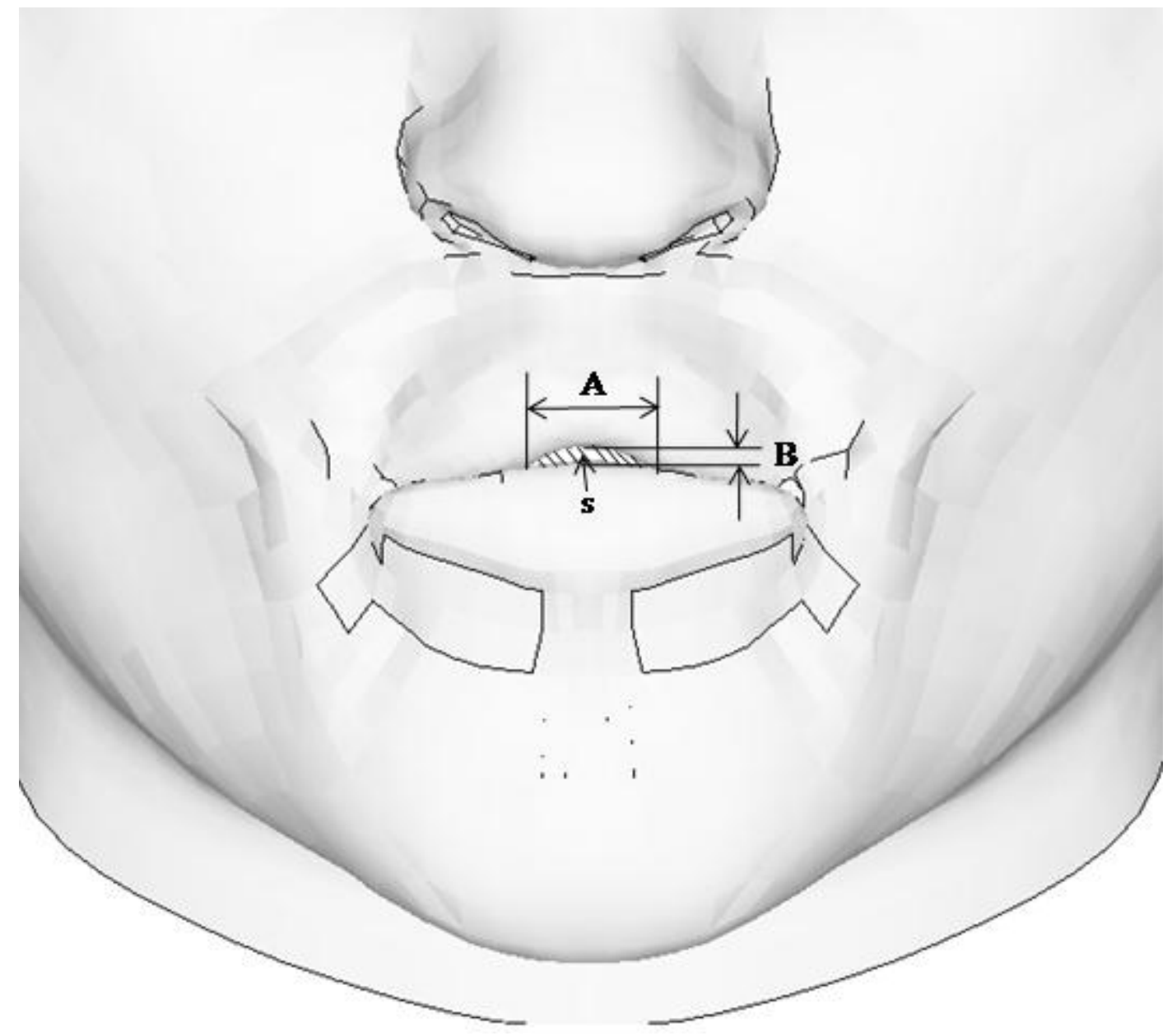

Figure 8- Lip shape parameters (frontal view): lip area (S), lip width (A), lip height (B) (Abry \& Boë, 1986). 


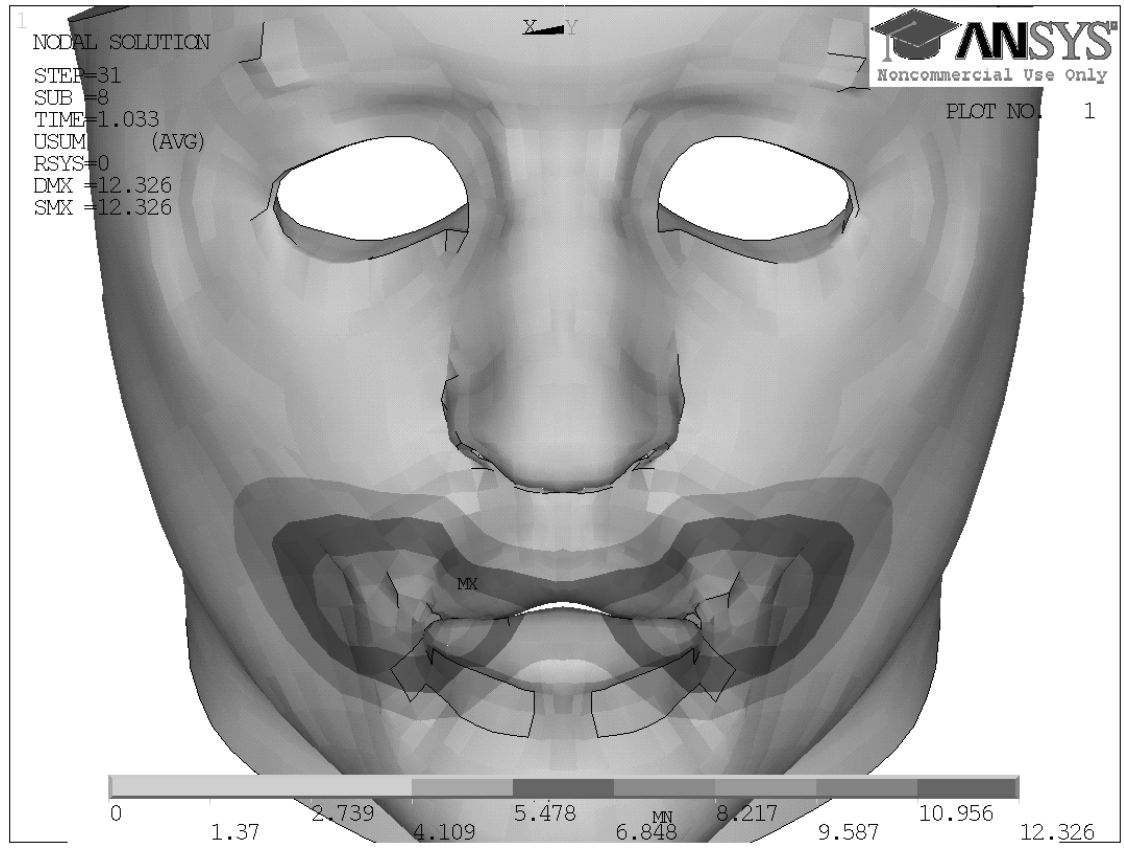

(a)

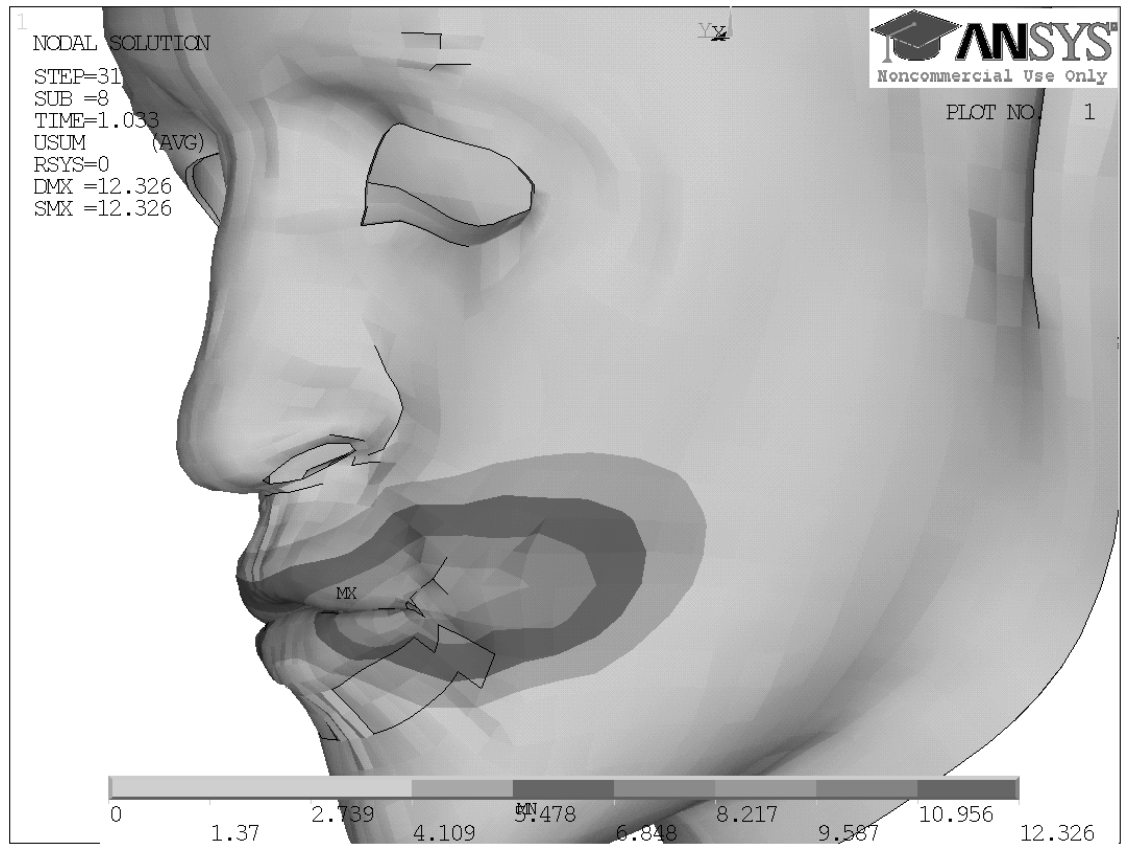

(b)

Figure 9- OOP activation with maximal stiffening: (a) front view, (b) profile view. 


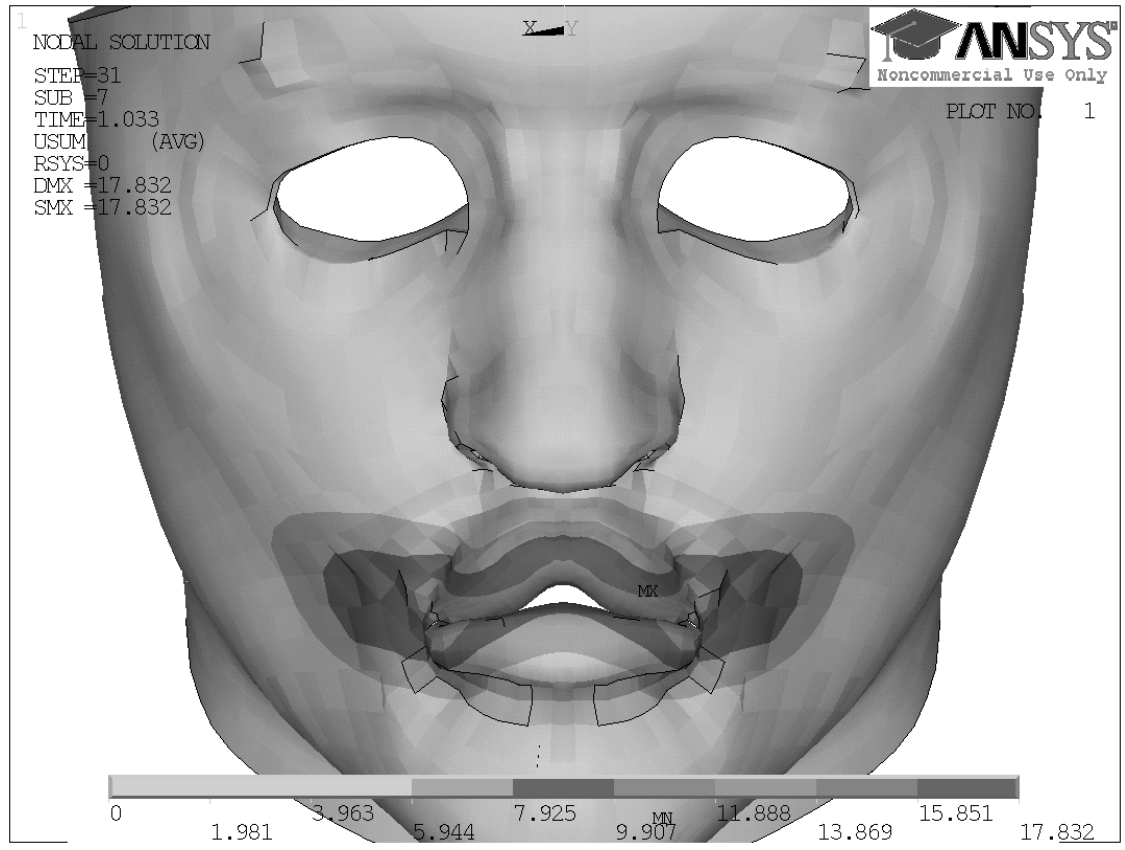

(a)

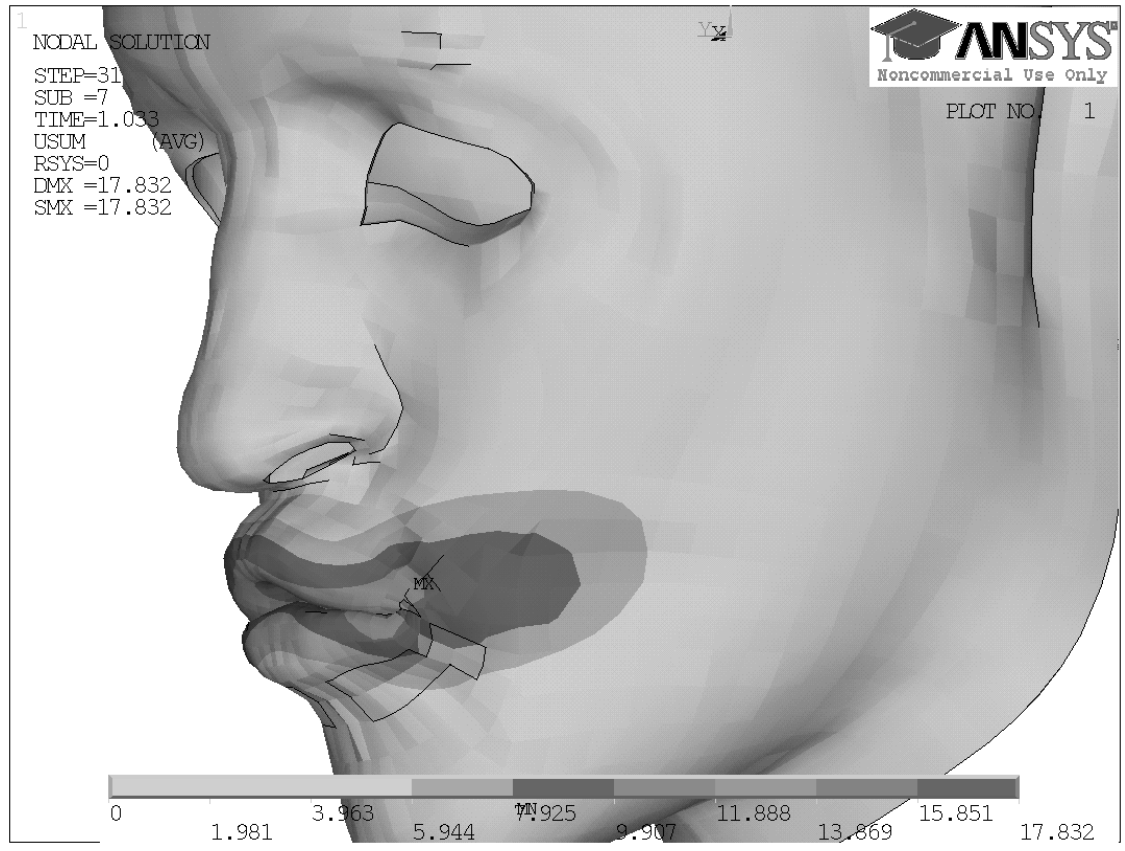

(b)

Figure 10- OOP activation without stiffening (same activation as in Figure 9): (a) front view, (b) profile view. 

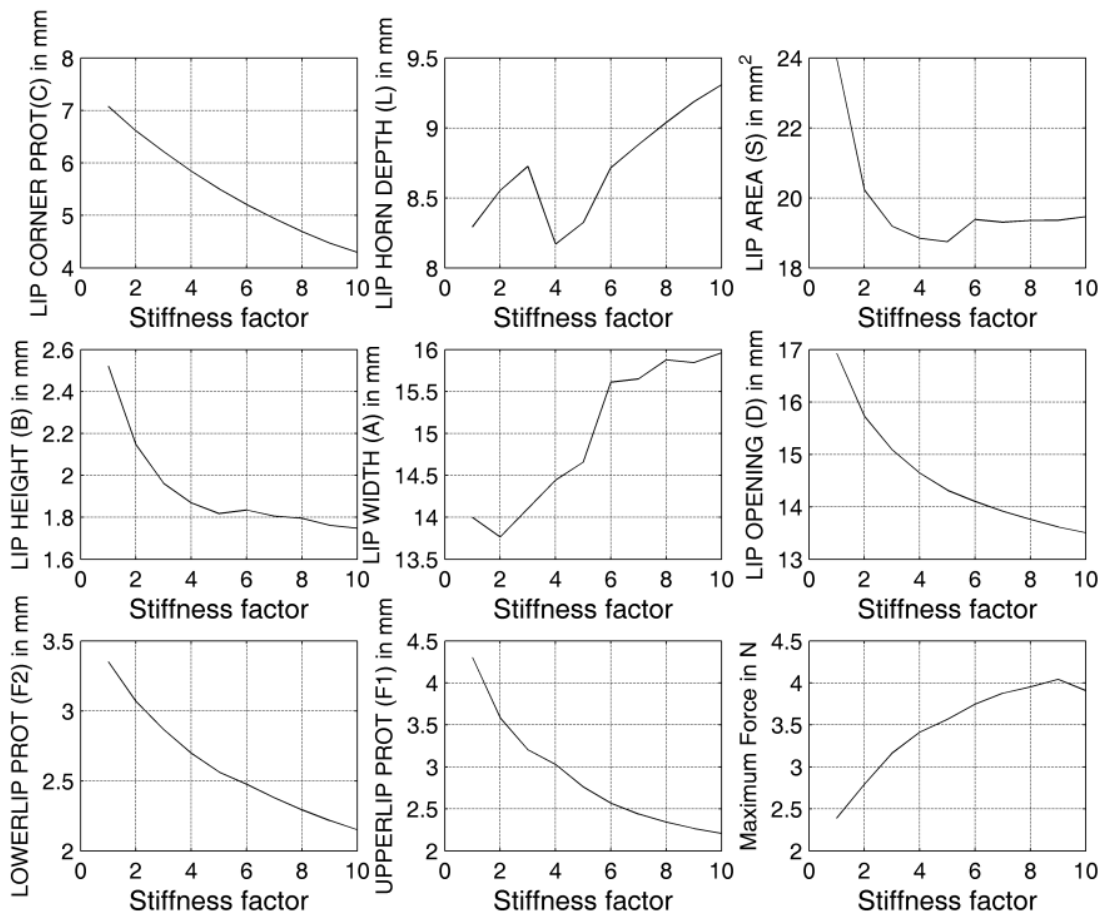

(a)
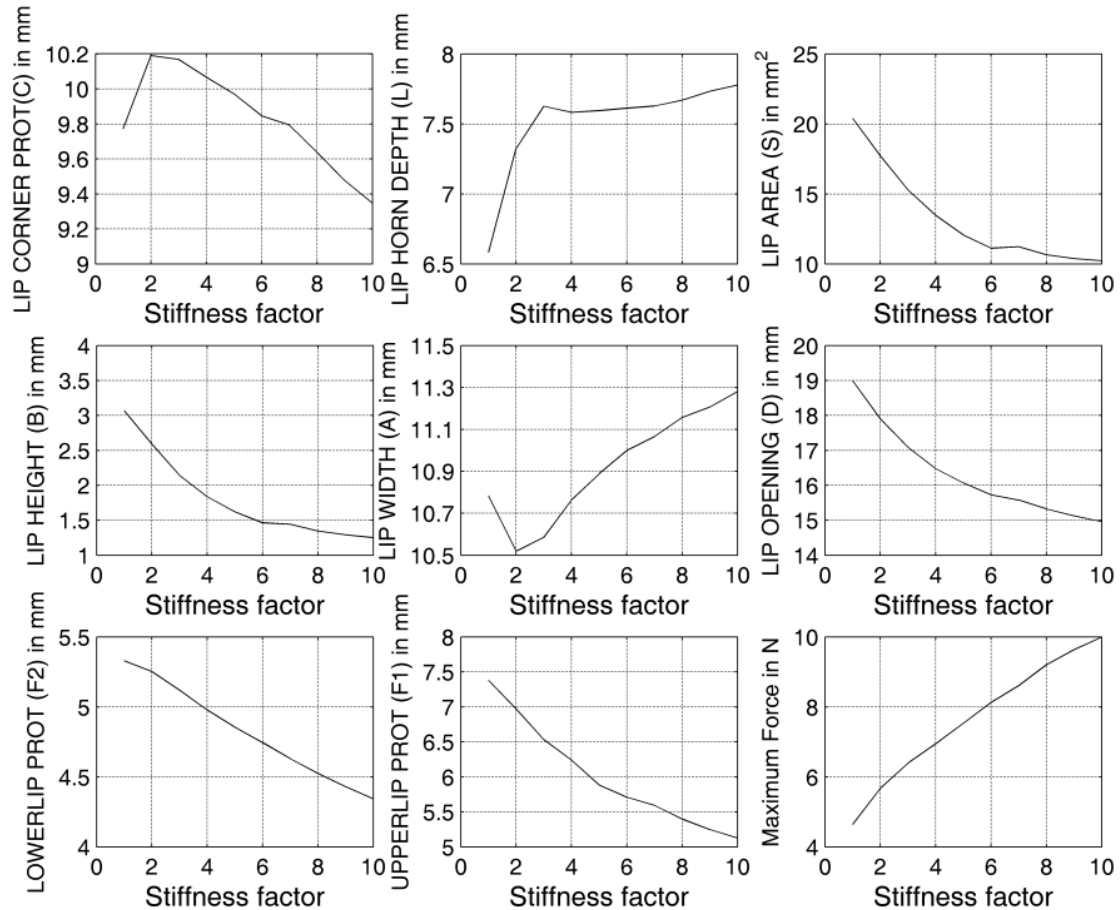

(b)

Figure 11- Variation in lip parameters as a function of stiffness: (a) with minimum activation, and (b) with maximum activation. 

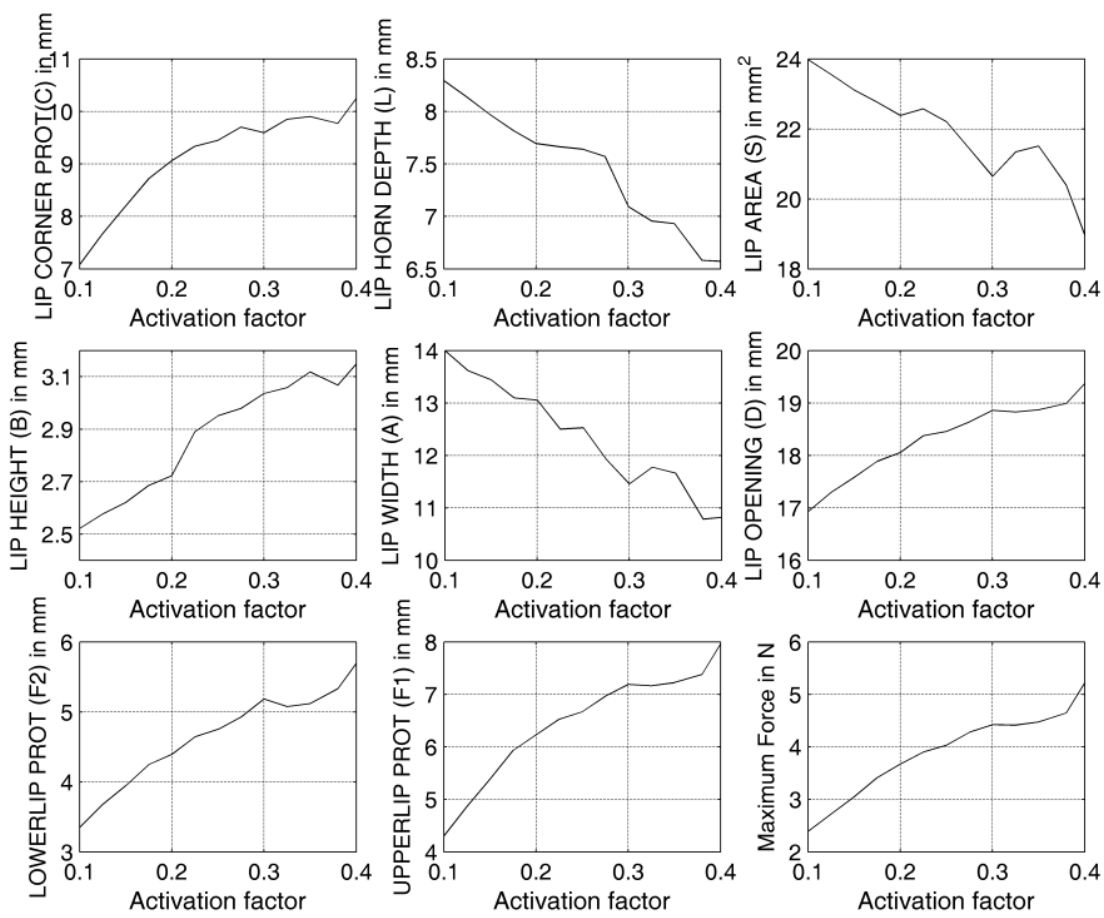

(a)
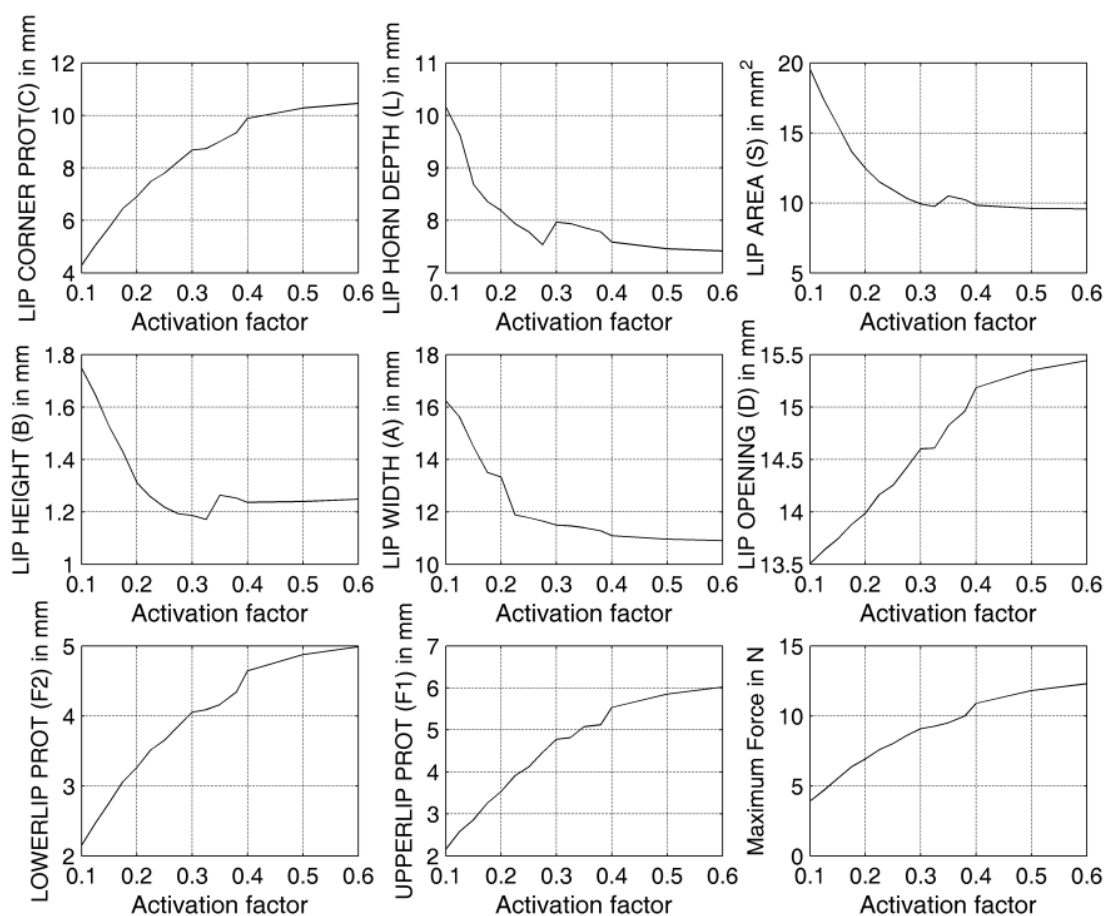

Activation factor

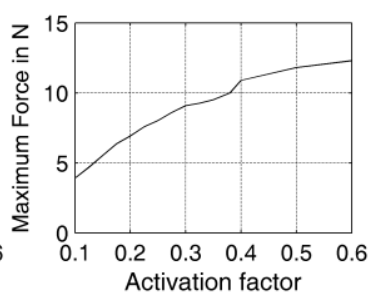

(b)

Figure 12- Variation in lip parameters as a function of activation with (a) no stiffness change, (b) maximum stiffness change. 


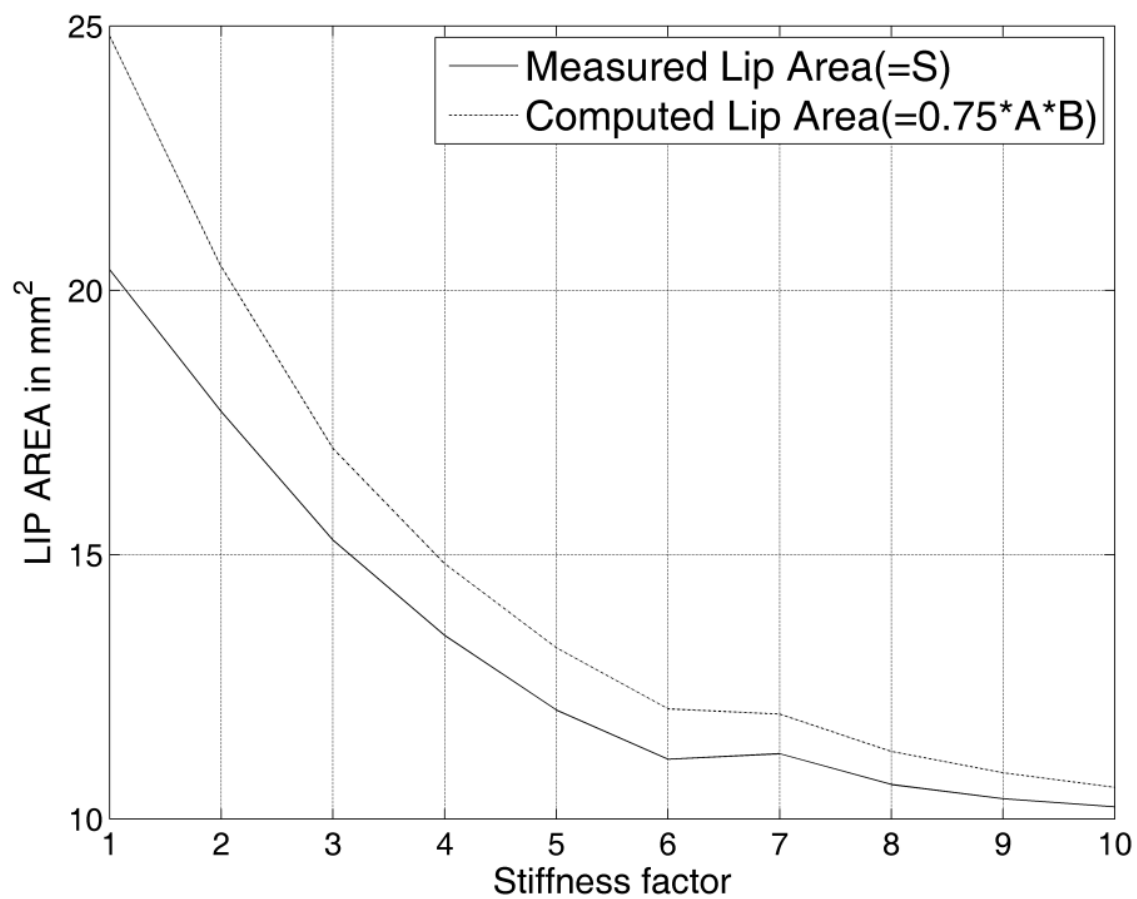

(a)

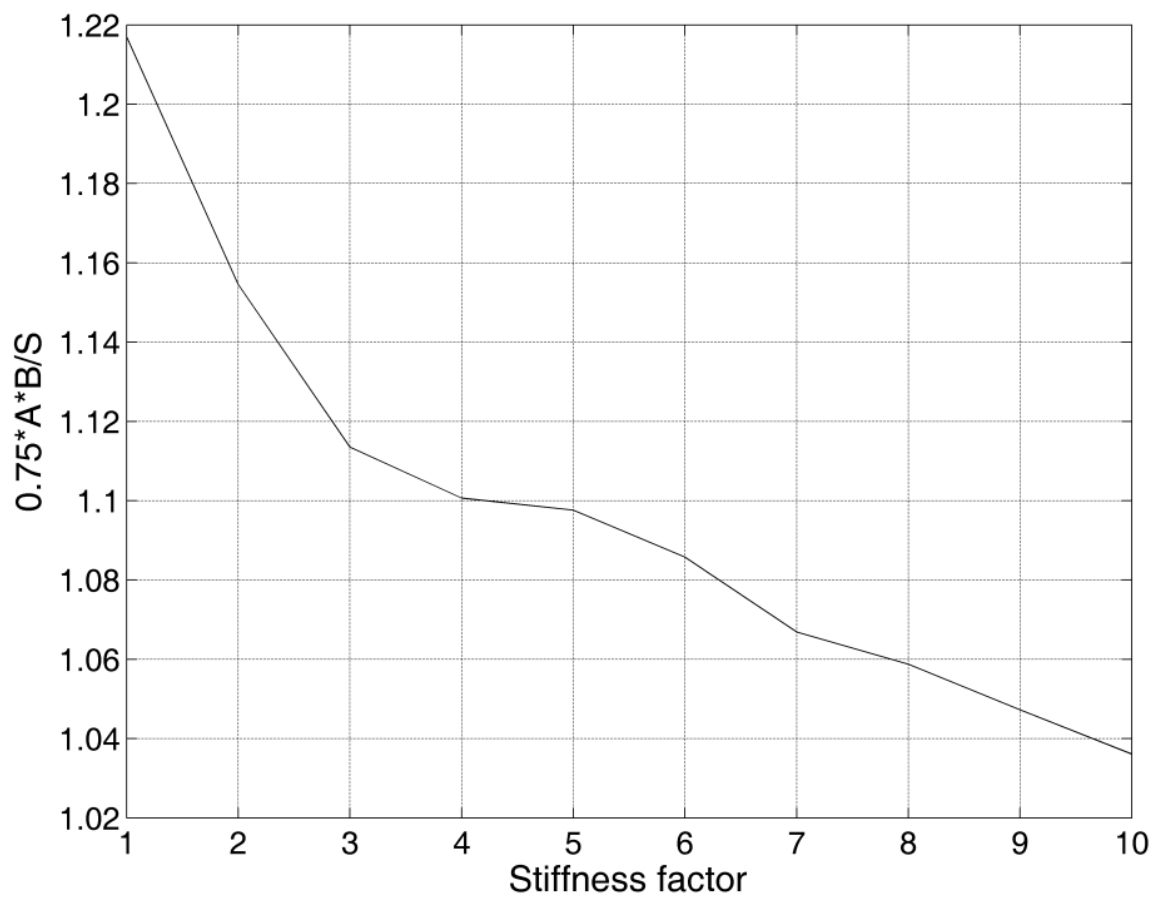

(b)

Figure 13- Lip area as a function of stiffness: (a) calculated using Abry \& Boë's formula (dashed line) and measured from our simulations (solid line), (b) the ratio of calculated to measured area. 


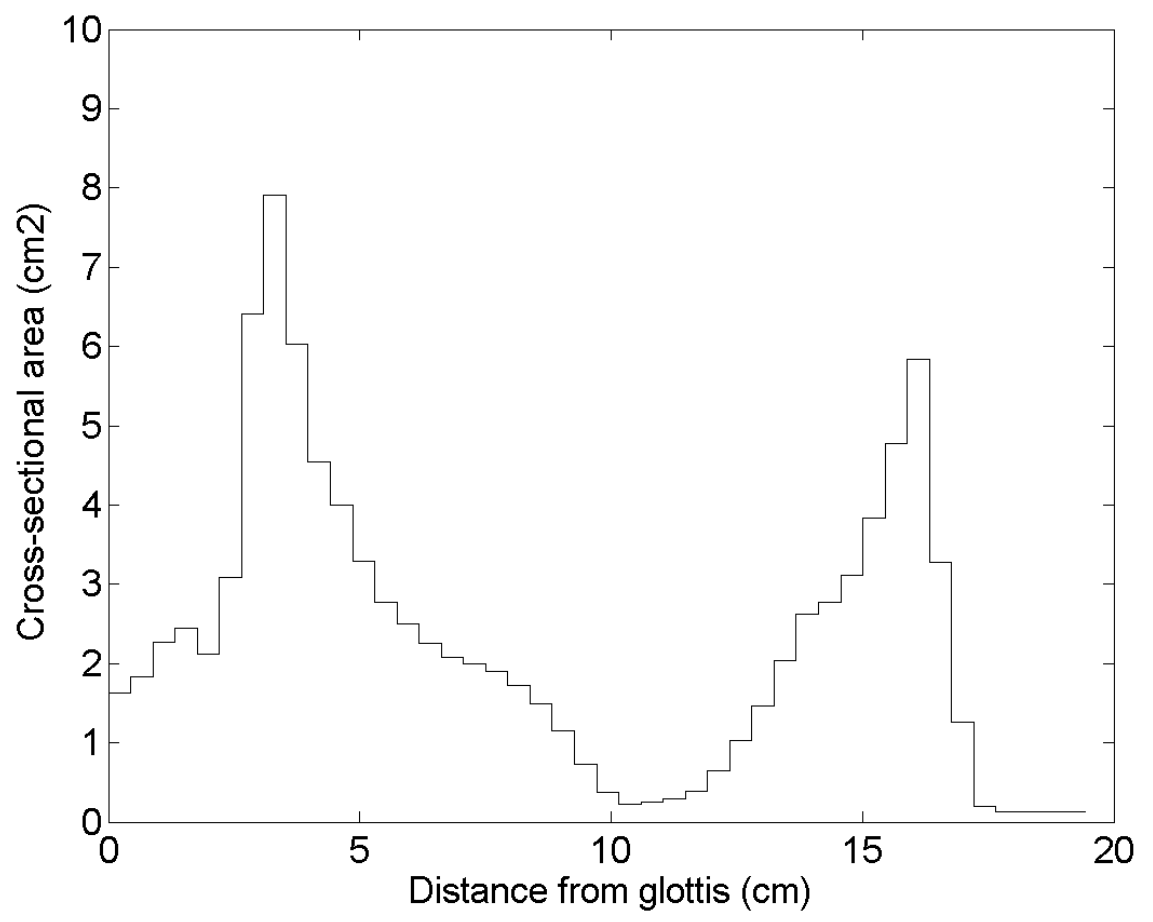

(a)

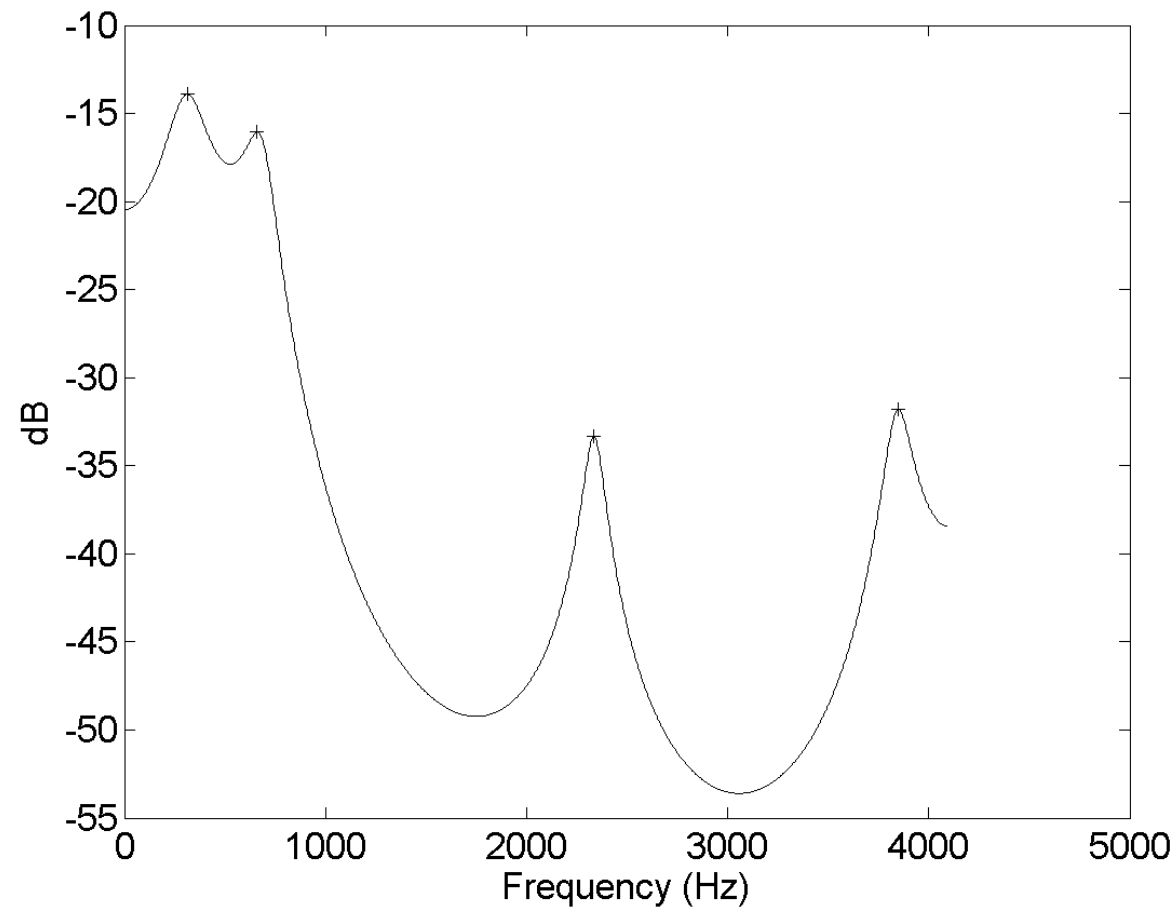

(b)

Figure 14- Study of spectral properties of a synthetic French vowel /u/: (a) an example of area function, (b) the spectral envelope of a signal produced from this area function computed using the Linear Prediction Analysis (the crosses show the speech formants). 


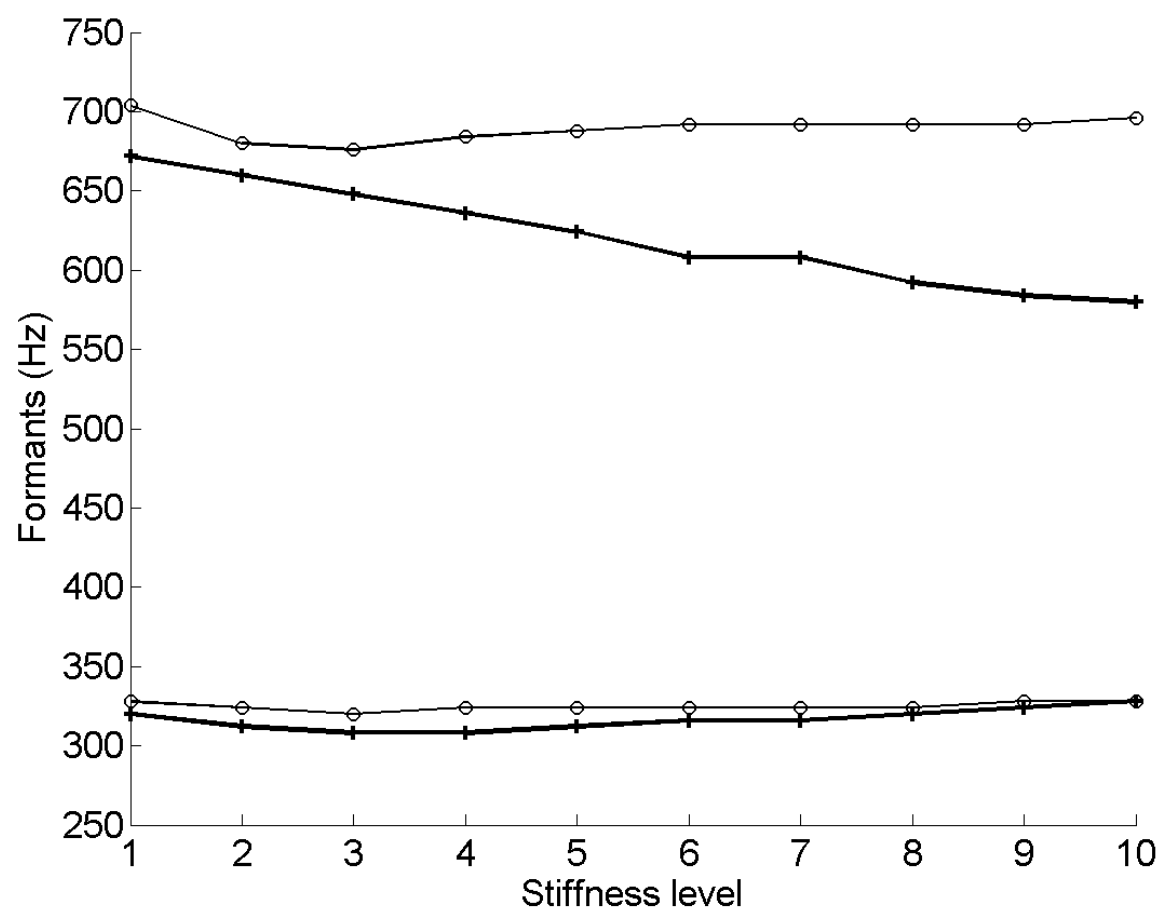

(a)

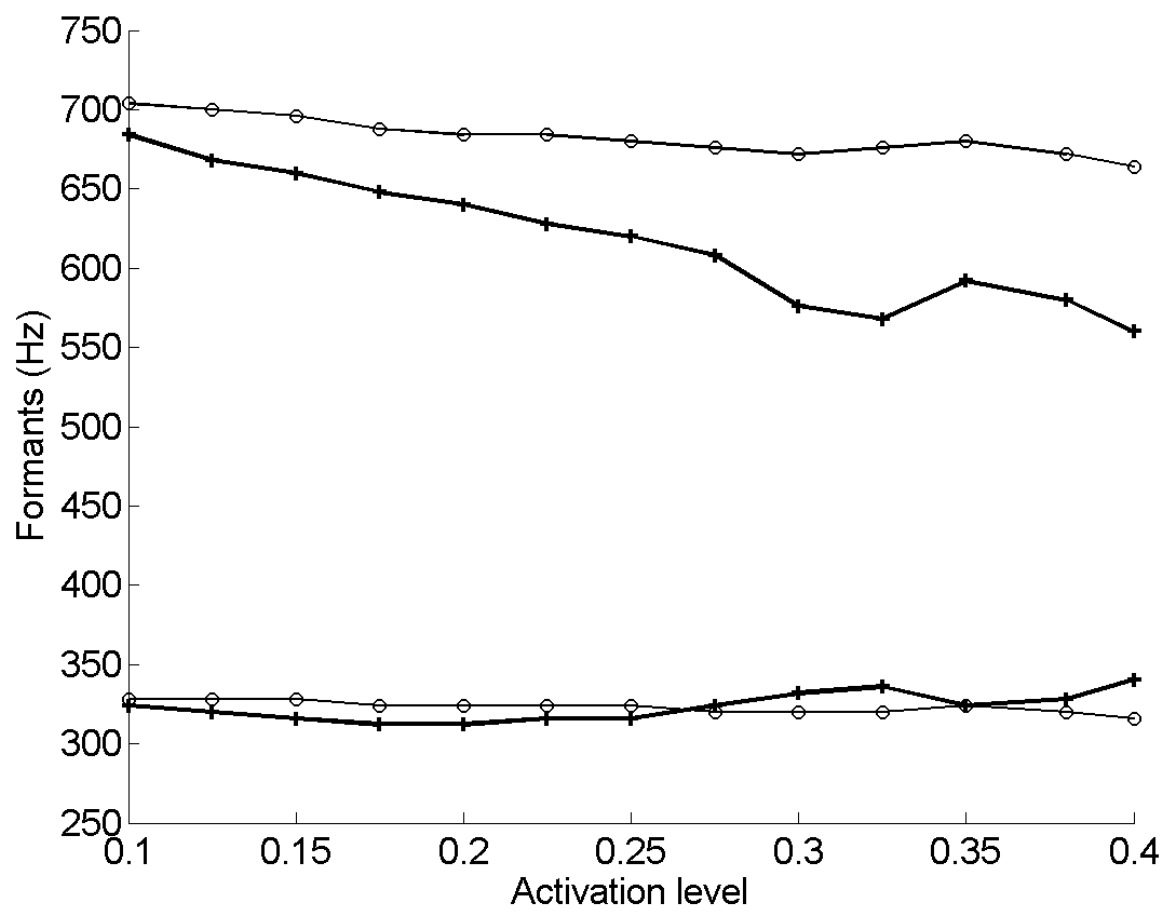

(b)

Figure 15- The variation in the first two speech formants (F1 and F2) corresponding to the French vowel / $\mathrm{u}$ / for different (a) stiffness levels (the bold lines correspond to maximum activation levels) and (b) activation levels (the bold lines correspond to maximum stiffening values). 